\title{
Price, Volatility and the Second-Order Economic Theory
}

\author{
Victor Olkhov* \\ TVEL, Moscow, Russia
}

\section{ARTICLE INFO}

\section{Article history:}

Received 21 April 2021

Revised 19 June, 14 July 2021 and 12

August 2021

Accepted 25 August 2021

Published 30 August 2021

Keywords:

Price probability

Volatility

Economic theory

Market trades

Expectations

\begin{abstract}
We introduce the new price probability measure, which entirely depends on the probability measures of the value and the volume of the market trades. We define the nth statistical moment of the price as the ratio of the nth statistical moment of the value to the nth statistical moment of the volume of all trades performed during an averaging time interval $\Delta$. The set of the price statistical moments determines the price characteristic function and its Fourier transform defines the price probability measure. The price volatility depends on the 1st and the 2nd statistical moments of the value and the volume of the trades. The prediction of the price volatility requires a description of the sums of squares of the value and the volume of the market trades during the interval $\Delta$ and we call it the second-order economic theory. To develop that theory, we introduce numerical continuous risk ratings and distribute the agents by the risk ratings as coordinates. Based on distributions of the agents by the risk coordinates, we introduce a continuous economic media approximation that describes the collective trades. The agents perform the trades under the action of their expectations. We model the mutual impact of the expectations and the trades and derive equations that describe their evolution. To illustrate the benefits of our approach, in a linear approximation we describe perturbations of the mean price, the mean square price and the price volatility as functions of the first and the second-degree trades' disturbances.
\end{abstract}

\section{Introduction}

Price and volatility are the core issues of financial economics, and their diverse and versatile studies have a long and endless history. As a brief illustration of the breadth price and volatility investigations over nearly a century, we refer in précis notes to the papers by Hall and Hitch (1939), who assess how agents make the trade price decisions, Fama (1965) develops the random walk theory of stock prices, Stigler and Kindahl (1970) model the price volatility and begin their article with an important and very clear statement that should be repeated by all researchers: "The Unique Price, as we observed, is a myth". Pearce (1983) reviews the stock market and prices as leading indicators of economic

This research received no support, specific grant or financial assistance from TVEL or funding agencies in the public, commercial or nonprofit sectors. We welcome funding our studies.

* Corresponding author.

E-Mail address: victor.olkhov@gmail.com

ORCID: 0000-0003-0944-5113 
activity and business cycles; Tauchen and Pitts (1983) assess the joint probability distribution of the price and the change in the volume of transactions; Schwert (1988) examines the relations between the stock volatility, the real and nominal macroeconomic volatility, the stock trading activity and default risk using data for more than a century. Mankiw, Romer and Shapiro (1991) study the predictability of the stock prices; Campbell et.al. (1993) describe the relationship between the aggregate market trading volume and the serial correlation of daily stock returns; Heston (1993) generalized the Black-Scholl model and derived an option price solution for an asset with a stochastic volatility. Brock and LeBaron (1995) study the problem of whether volatility persistence comes from the fundamental value that the trading process is pricing or whether it comes from the trading process itself. Bernanke and Gertler (2000) examine the impact of the asset price volatility on inflation and central bank monetary policy; Andersen et.al. (2001) use direct, model-free measures of the daily volatility of the stock return and correlation obtained from the high-frequency intraday transaction prices on individual stocks and Andersen et.al. (2005) survey theoretical developments and empirical insights with a distinct focus on forecasting applications and discuss volatility forecast methods. Engle and Patton (2001) outline what should be incorporated in a volatility forecasting models; Poon and Granger (2003) provide a review of the volatility forecasting based on the time-series, conditional variance and on option of Implied Standard Deviation; Daly (2008) explains in non-technical terms various techniques used to measure the volatility; Christiansen, Schmeling and Schrimpf (2012) investigate, whether the asset return volatility is predictable by the macroeconomic and financial variables and consider the economic drivers of financial volatility; Padungsaksawasdi and Daigler (2018) introduce and empirically examine a new measure of realized volatility that includes the volume associated with the price of each trade. They refer to it as the volume weighted volatility (VWV) or, alternatively, demand-based volatility, Bogousslavsky and Collin-Dufresne (2019) examine the relation between the liquidity, volume, and volatility using a comprehensive sample of U.S. stocks. This is only a small part of the infinite number of the important and complex studies of the price-volatility problems. We refer to them to show that almost all the factors, models and relations concern the price and the volatility origin, dynamics, measurements, forecasts and impact on the financial markets and economic development have already been under investigation for decades.

Nevertheless, we propose a fresh look on the price-volatility problem. We introduce the new price probability measure that is entirely determined by the random properties of market trades. We show that any predictions of the average price, price volatility or price probability measure require a forecast of the properties of market trading. We underline that any description of the random financial markets should model the economic and financial variables aggregated during certain averaging time interval $\Delta$. Any economic averaging procedure presupposes that particular time-series of the financial variables be aggregated during some interval $\Delta$. This averaging interval $\Delta$ can be equal to a minute, an hour, a day, a month, etc. Any description of the mean price and the price volatility uses financial data aggregated during certain averaging interval $\Delta$. The choice of the interval $\Delta$ and the choice of the aggregation method of the economic and financial data, the price time-series, trade, investment, credits, profits time-series, etc., plays a core role for economic modeling and forecasting.

Modern macroeconomic and financial theories describe the evolution of the first-degree macro variables aggregated during a certain interval $\Delta$. For example, macro investment, credits and assets are determined as the sums 
of investment, credits and assets of the economic agents (without duplication) aggregated during the time interval $\Delta$. The sums of trades during the interval $\Delta$ define macro trades; the sums of consumption, taxes and debts define total macro consumption, taxes and debts in the economy aggregated during the interval $\Delta$. All these macro variables are defined as the sums of the first-degree variables. We call the description of the first-degree variables as the first-order economic theory. Almost all macroeconomic and financial variables are the first-degree variables.

The price volatility is almost the only exception. Indeed, the price volatility describes difference between the mean square price and the square of the mean price. The price probability measure that determines the mean price, the mean square price and the price volatility is the origin of the important problems. Actually, investors and researchers use different definitions of the mean price.

The most conventional way to define the price probability measure $\mathrm{p}$ and assess the mean price and the price volatility during the interval $\Delta$ is based on the implicit assumption that all $\mathrm{N}$ market trades performed during the averaging interval $\Delta$ have equal probability $1 / \mathrm{N}$ and hence, the probability $\mathrm{P}(\mathrm{p})$ of the price $\mathrm{p}$ of a single trade is equal to $\mathrm{P}(\mathrm{p})=1 / \mathrm{N}$. If there are $\mathrm{np}$ trades at price $\mathrm{p}$ during the averaging interval $\Delta$, then the probability $\mathrm{P}(\mathrm{p})$ of the price $\mathrm{p}$ and the mean price $\mathrm{E}(\mathrm{p})$ during the interval $\Delta$ take form

$$
P(p)=\frac{n_{p}}{N} \quad ; \quad E(p)=\sum p_{k} P\left(p_{k}\right)=\frac{1}{N} \sum p_{k} n_{p_{k}} ; \quad N=\sum n_{p_{k}}
$$

The relations (1.1) are so common and so simple that almost no additional considerations are taken to support (1.1). During the last decades, however, a different definition of the mean price has been proposed. The volume weighted average price (VWAP) $p(1 ; t)$ was introduced and studied by (Berkowitz et.al 1988; Buryak and Guo, 2014; Guéant and Royer, 2014; Busseti and Boyd, 2015; Padungsaksawasdi and Daigler, 2018, CME Group, 2020).

$$
\begin{gathered}
C(1 ; t)=\sum_{i=1}^{N(t)} C\left(t_{i}\right) \quad ; \quad U(1 ; t)=\sum_{i=1}^{N(t)} U\left(t_{i}\right) ; C\left(t_{i}\right)=p\left(t_{i}\right) U\left(t_{i}\right) \\
p(1 ; t)=\frac{1}{U(1 ; t)} \sum_{i=1}^{N(t)} p\left(t_{i}\right) U\left(t_{i}\right)=\frac{C(1 ; t)}{U(1 ; t)} \\
\Delta(t)=\left[t-\frac{\Delta}{2}, t+\frac{\Delta}{2}\right] ; t_{i} \in \Delta(t), i=1, \ldots N(t)
\end{gathered}
$$

Here, $\mathrm{C}(\mathrm{ti})$ denotes the value and $\mathrm{U}(\mathrm{ti})$ the volume (1.2) of a trade at price $\mathrm{p}(\mathrm{ti})$ performed at the moment ti during the averaging interval $\Delta(\mathrm{t})(1.4)$. VWAP defines the mean price $\mathrm{p}(1 ; \mathrm{t})$ as the ratio (1.3) of the sum of the values $\mathrm{C}(\mathrm{ti})$ to the sum of the volumes $\mathrm{U}(\mathrm{ti})$ of all $\mathrm{N}(\mathrm{t})$ market trades during the interval $\Delta(\mathrm{t})$.

The collision between two definitions of the mean price hides the difference between two approaches to describe the irregular price evolution. The conventional approach defines the price probability $\mathrm{P}(\mathrm{p})(1.1)$ as the ratio of frequency $n p$ of trades at price $\mathrm{p}$ to total number of trades $\mathrm{N}$ during the interval $\Delta(\mathrm{t})$ and use it to describe the mean price, volatility, etc. Relations (1.1) implicitly assumes that a transaction with $t$ the market trades values and volumes he trade value $\$ 1$ has the same probability and the same impact on the mean price as a transaction with the trade value $\$ 1$ bln, and that seems a bit economically inconsistent. Investors prefer foresee the price trends driven by the market dynamics. The VWAP $(1.2 ; 1.3)$ takes into account the economic value, the market impact of the trade volume U(ti) 
at price $\mathrm{p}(\mathrm{ti})$. The description of the mean price via the frequency-based price probability (1.1) that has a little to do with the market time-series is complemented or replaced by the description of the mean price as VWAP $(1.2 ; 1.3)$ that is determined by the sum of the values $\mathrm{C}(\mathrm{ti})$ and volumes $\mathrm{U}(\mathrm{ti})$ of market trades during $\Delta$.

Let us outline the structural guideline of our paper to help readers follow our reasoning and understand the results. In Section 2, we consider the economic sense of the frequency-based price probability (1.1) and VWAP (1.2) and introduce the new price probability measure $\eta(p ; t)$ that entirely depends on the statistical properties of the market trades. In Section 2, we explain how the set of the price statistical moments determines the price characteristic function $F(x ; t)$ and the price probability measure $\eta(p ; t)$. The direct dependence of the price characteristic function $\mathrm{F}(\mathrm{x} ; \mathrm{t})$ and the price probability measure $\eta(\mathrm{p} ; \mathrm{t})$ on the random properties of the market trades values and volumes uncovers the complex impact of the randomness of market trading on stochastic price dynamics and explains the complexity of price forecasting. For example, the price volatility depends on the mean square of the price $p(2 ; t)$, and thus, the price volatility is determined by the aggregated squares of the value $\mathrm{C}(2 ; \mathrm{t})$ and the volume $\mathrm{U}(2 ; \mathrm{t})$. Hence, the prediction of the price volatility requires the forecast of the sums of squares of the trades $(\mathrm{U}(2 ; \mathrm{t}) ; \mathrm{C}(2 ; \mathrm{t}))$, and that needs the development of a second-order economic theory - Section 3. To avoid any misunderstanding, we emphasize that we introduce the price probability measure and describe the stochastic price framework without any use of notions, terms or methods of the so-called "quantum probability", "quantum price" models, etc.

In Section 3, we consider the description of the sums of squares of values and volumes of trades and start studying the second-order economic theory. It is obvious that the mean square price $p(2 ; t)$ and the price volatility are not the only second-degree variables. All macro variables such as bank rates, inflation, currency exchange rates, return on investment, GDP growth rates, etc. fluctuate and their volatilities are described by the aggregated squares, similar to the price volatility. We do not describe here the volatility of bank rates, inflation or GDP, but underline that description of these problems is similar to description of the price volatility via the second-order economic theory. Description of the price volatility and the sums of squares of values and volumes of trades is only a special case of the second-order theory, which models the evolution and interactions of squares of the economic variables of agents aggregated during the interval $\Delta$. We leave the general treatment for the future and consider in Section 3 description of the aggregated squares of market trades. We use the methods developed in (Olkhov, 2016a-2017a; 2018-2020) and introduce a continuous media approximation that describes the evolution and interactions of the aggregated squares of value and volume.

It is well known that agents perform their market trades under the action of their expectations. Thus, description of the trades should depend on the agents' expectations. On the other hand, the executed market trades determine the agents' expectations. The mutual interactions between the trades and the expectations establish rather a complex system. In Sec 4, we introduce the main notions of the collective expectations, which affect trades and derive equations describing mutual interactions and dynamics of the trades and the expectations. Such long considerations uncover complex relations that govern the evolution of the sums of squares of the values and volumes of trades under the action of the collective expectations. 
To illustrate the benefits of our approach to the price volatility problem, in Section 5 we consider a special case and describe, in a linear approximation by perturbations, the disturbances of the mean price, mean square price and price volatility as functions of the second-degree trading disturbances. Section 6 - Conclusion.

We collect most of the calculations and equations in Appendices A-D. In Appendix A., we consider the economic continuous media approximation of the sums of squares of the values and volumes of the trades. In Appendix B, we introduce definitions of the collective expectations. In Appendix C, we derive equations on the second-degree trades. Appendix D describes perturbations of the mean square price and the price volatility.

We believe that the mathematical complexity of our methods corresponds to the complexity of the price volatility problem. However, readers may choose only particular sections to get main ideas and reasons that support our model. Readers may chose only Section 2 to get the main considerations about the price probability measure, mean price and price volatility problem described as functions of the market trades. Those who read Sections 3 and 4 may learn the basic notions of the second-order economic theory that models the evolution of the sums of squares of values and volumes of trades and the collective expectations. Section 5 presents a linear approximation that describes perturbations of the mean square price and the price volatility.

Equations (3.4) denote Section 3 and equation 4. We use roman letters A, B, d to denote scalars and bold $\boldsymbol{B}, \boldsymbol{P}, \boldsymbol{v}$ - to denote vectors. We assume that readers are familiar with basic notions and methods of stochastic dynamic systems, probability density functions, statistical moments and characteristic functions, and refer (Klyatskin, 2005; 2015) for details. We hope that the usual notations for the partial differential equations do not require clarifications.

\section{Price and volatility}

In this section, we consider the reasons in favour of the new price probability measure. We define the nth statistical moment of price as the ratio of the nth statistical moment of value to the nth statistical moment of volume determined during the averaging interval $\Delta$. The sets of all statistical moments of value, volume and price determine characteristic functions of value, volume and price, and thus probability measures of value, volume and price.

Price is one of the most conventional and fundamental notions in economics and finance. Any price and volatility forecasts should operate with a certain price probability measure. The origin and economic justification of the price probability measure reflect one of the most important issues in modern finance. The most conventional treatment of market price probability is determined by (1.1). A simple but implicit assumption that any trade event, i.e., any trade performed during the averaging interval $\Delta$, has the equal probability defines the origin of the frequency-based price probability. If $N$ trades were performed during interval $\Delta$, then the probability of a single trade is equal to $1 / N$. If $n_{p}$ trades have the same price $p$, then the probability $P(p)$ of price $p$ is equal to $n_{p} / N$.

As usual most conventional issues hide most of the miracles and complexity. The miracles concern the total prevalence of the obvious and simple frequency-based price probability measure (1.1). We are not going to refute or disproof such conventional treatment of price probability. Economics is a social science and most financial issues exist due to the agents' preferences, tastes and expectations. Investors chose notions, models and methods on their own and then take financial decisions that lead to market trades and economic transactions. The most influential factor affecting the agents' preferences is the value of expected returns. Thus, any miracle story that convinces an 
investor of the reliability and profitability of a proposed model, forecast or definition may succeed and may become a crucial factor in the markets.

Instead of confronting the conventional price probability (1.1) we propose to have a different look on the meaning of the market price, price probability and the statistical moments of price. We take the trivial definition of the price $p\left(t_{i}\right)$ at the moment $t_{i}$ as the coefficient between the value $C\left(t_{i}\right)$ and the volume $U\left(t_{i}\right)$ of market trade:

$$
C\left(t_{i}\right)=p\left(t_{i}\right) U\left(t_{i}\right)
$$

Investors and economic agents perform trades under the action of their personal expectations. Investors may take any arbitrary price model and adopt any reasons that support their decisions to execute the market trade at a particular price (2.1). For sure, agents' expectations impact the price $p$ (2.1). However, the value $C\left(t_{i}\right)$ and the volume $U\left(t_{i}\right)$ of a particular trade (2.1) determine price $p\left(t_{i}\right)$. Thus, we believe that describing the random properties of price timeseries by the conventional frequency-based price probability (1.1), which does not take into account the randomness of the values and volumes of the market trades, seems to be odd and uncountable. Price stochasticity cannot be treated independently of the randomness of market trading. Market trades determine the price randomness, and hence the statistical properties of value and volume should affect the statistical properties of price.

As we already mentioned, more than 30 years ago Berkowitz et.al (1988) introduced the volume weighted average price (VWAP) and this way to measure the mean price $p(1 ; t)(1.2 ; 1.3)$ becomes a very common (Buryak and Guo, 2014; Guéant and Royer, 2014; Busseti and Boyd, 2015; Padungsaksawasdi and Daigler, 2018, CME Group, 2020). We take the VWAP $(1.2 ; 1.3)$ as a starting point and introduce the new price probability measure $\eta(p ; t)$ that determine all price statistical moments similar to $(1.2 ; 1.3)$.

We consider a probability measure $\eta(p ; t)$ of price $p$ at the moment $t$ that describes the statistical price properties of the market trades performed during the averaging interval $\Delta(t)(1.4)$. Let us take that during the interval $\Delta(t)$ there were performed $N(t)$ market trades at prices $p_{i}, i=1,2, . . i(N)$. The price probability measure $\eta(p ; t)$ defines the price $n t h$ statistical moment or the mean $n t h$ power of price $p(n ; t)$ at moment $t$ as:

$$
p(n ; t)=\sum_{i=1}^{i(N)} p_{i}^{n} \eta\left(p_{i} ; t\right) \quad ; n=1,2, \ldots
$$

It is well known that probability measure defines all statistical moments (2.2) and the same time the set of statistical moments $p(n ; t)$ (2.2) entirely defines the probability measure $\eta(p ; t)(2.2)$ through its characteristic function (Shephard, 1991; Shiryaev, 1999; Klyatskin, 2005; Klyatskin, 2015). To define the probability measure $\eta(p$; $t$ ) it is enough to define price statistical moments $p(n ; t)$ for all $n=1,2, \ldots$.

To define the set of price statistical moments $p(n ; t)$ we start with trivial relation (2.1) and indicate that the same valid for any $n t h$ power of price $p^{n}\left(t_{i}\right)$ :

$$
C^{n}\left(t_{i}\right)=p^{n}\left(t_{i}\right) U^{n}\left(t_{i}\right) \quad ; \quad n=1,2, \ldots
$$


We underline that the definition of VWAP $(1.2 ; 1.3)$ implies that the random volume $U(t i)$ does not correlate with the price $p(t i)$. Let us denote $\mathrm{E}[.$.$] as mathematical expectation and due to (1.3)$

$$
C(1 ; t)=E\left[C\left(t_{i}\right)\right]=E\left[p\left(t_{i}\right) U\left(t_{i}\right)\right]=E\left[p\left(t_{i}\right)\right] E\left[U\left(t_{i}\right)\right]=p(1 ; t) U(1 ; t)
$$

We define the $n t h$ statistical moment $(2.2)$ of the price $p(n ; t)$ as:

$$
C_{m}(n ; t)=E\left[C^{n}\left(t_{i}\right)\right]=E\left[p^{n}\left(t_{i}\right) U^{n}\left(t_{i}\right)\right]=E\left[p^{n}\left(t_{i}\right)\right] E\left[U^{n}\left(t_{i}\right)\right]=p(n ; t) U_{m}(n ; t)
$$

and note that our definition (2.4) implies that nth power of the volume $U n(t i)$ does not correlate with nth power of the price $p n(t i)$. In (2.4) the nth statistical moments of the value $\mathrm{Cm}(n ; t)$ and the volume $U m(n ; t)$ of $N(t)$ trades during the averaging interval $\Delta(t)(1.4)$ have the conventional form (2.5):

$$
C_{m}(n ; t)=\frac{1}{N(t)} \sum_{i=1}^{N(t)} C^{n}\left(t_{i}\right) ; \quad U_{m}(n ; t)=\frac{1}{N(t)} \sum_{i=1}^{N(t)} U^{n}\left(t_{i}\right)
$$

The relations (2.5) presuppose that for $\mathrm{N}$ trades performed at moments $t i, i=1,2, . . N$ during the averaging interval $\Delta$ probability $P(t i)$ of a single trade at moment ti equals $1 / \mathrm{N}$. That assumption determines the conventional frequencybased probabilities measures of the value $v(C ; t)$ and the volume $\mu(U ; t)$

$$
P\left(t_{i}\right)=\frac{1}{N} \quad ; \quad v(C ; t)=\frac{n(C)}{N} \quad ; \quad \mu(U ; t)=\frac{n(U)}{N}
$$

$n(C)$-number of trades at value $C$ and $n(U)$ - number of trades at volume $U . P\left(C\left(t_{i}\right)\right)$ and $P\left(U\left(t_{i}\right)\right)$ - probability of trade at moment $t_{i}$. Actually, (2.6) determines the ground, the approach to frequency-based definition of the probability measures of the value and the volume. It is obvious, that the probability measures itself are determined by the time-series of the market trades those define the frequencies $n(C)$ and $n(U)$ for the trades performed during the averaging interval $\Delta$. We underline that (2.4) does not imply statistical independence of the random volume $U\left(t_{i}\right)$ and price $p\left(t_{i}\right)$. Definition of the statistical moment of the price $p(n ; t)(2.4)$ implies no correlations between $p^{n}\left(t_{i}\right)$ and $U^{n}\left(t_{i}\right)$ only. It is easy to show (2.3-2.5) that any nth power of the volume $U^{n}\left(t_{i}\right)$ and $k t h$ power of the price $p^{k}\left(t_{i}\right)$ for $n \neq k$ correlates:

$$
E\left[p^{k}\left(t_{i}\right) U^{n}\left(t_{i}\right)\right] \neq p(k ; t) U_{m}(n ; t) \quad ; \quad k \neq n
$$

It is obvious that the normalizing factor $1 / \mathrm{N}$ in (2.5) does not impact (2.4). Hence, we can define the price statistical moment $p(n ; t)(2.2)$ as the coefficient (2.7) between the sum $C(n ; t)(2.7)$ of $n t h$ power of the values $C^{n}\left(t_{i}\right)$ and the sum $U(n ; t)(2.7)$ of $n t h$ power of the volumes $U^{n}\left(t_{i}\right)$ of trades during $\Delta(t)$ : 


$$
C(n ; t)=\sum_{i=1}^{N(t)} C^{n}\left(t_{i}\right) ; U(n ; t)=\sum_{i=1}^{N(t)} U^{n}\left(t_{i}\right) ; C(n ; t)=p(n ; t) U(n ; t)
$$

For $n=1$ the definition of the mean price $p(1 ; t)(2.4-2.7)$ completely coincides the VWAP $(1.2 ; 1.3)$. One can present $(2.4 ; 2.7)$ in the form similar to $(1.3)$ :

$$
p(n ; t)=\frac{1}{U(n ; t)} \sum_{i=1}^{N(t)} p^{n}\left(t_{i}\right) U^{n}\left(t_{i}\right)=\frac{C(n ; t)}{U(n ; t)}
$$

We avoid excessive discussion in favour of our definition of the price statistical moments (2.4-2.7) and turn to the description of interesting and complex consequences of that choice.

The expressions of the price statistical moments $p(n ; t)(2.2)$ through $(2.4-2.8)$ define the price probability measure $\eta(p ; t)(2.2)$. For simplicity we use a continuous representation for the price probability measure $\eta(p ; t)(2.2)$ and take the price statistical moment $p(n ; t)$ as:

$$
p(n ; t)=\int d p p^{n} \eta(p ; t) \quad ; \quad n=1,2, \ldots \quad ; \quad \int d p \eta(p ; t)=1
$$

The Fourier transform of the price probability measure $\eta(p ; t)(2.9)$ determines the price characteristic function $F(x ; t)$ and vice versa (2.10) (Shephard, 1991; Shiryaev, 1999; Klyatskin, 2005; 2015):

$$
F(x ; t)=\int d p \eta(p ; t) \exp (i x p) \quad ; \quad \eta(p ; t)=\int d x F(x ; t) \exp (-i x p)
$$

For brevity we omit in (2.10) the normalizing factors proportional to $(2 \pi)$. It is easy to show that the $n t h$ derivative of the price characteristic function $F(x ; t)(2.10)$ by variable $x$ at point $x=0$ gives the $n t h$ statistical moment $p(n ; t)$ :

$$
\left.\frac{d^{n}}{(i)^{n} d x^{n}} F(x ; t)\right|_{x=0}=\int d p p^{n} \eta(p ; t)=p(n ; t)
$$

The price characteristic function $F(x ; t)$ can be presented by Tailor series of (2.12) with statistical moments $p(n ; t)$ (2.4-2.8) (Klyatskin, 2005; Klyatskin, 2015):

$$
F(x ; t)=1+\sum_{i=1}^{\infty} \frac{i^{n}}{n !} p(n ; t) x^{n}
$$

We underline that the price statistical moments $p(n ; t)(2.4-2.8)$ determine the characteristic function (2.12).. However, (2.5) and the probability measures (2.6) define statistical moments of the value $C_{m}(n ; t)$ and the volume $U_{m}(n ; t)$. Probability measures (2.6) define statistical moments (2.5) and thus, define the characteristic functions of the value $G(x ; t)$ and the volume $Q(x ; t)(2.13)$ of random market trades during the interval $\Delta(1.4)$ :

$$
G(x ; t)=1+\sum_{i=1}^{\infty} \frac{i^{n}}{n !} C_{m}(n ; t) x^{n} \quad ; \quad Q(x ; t)=1+\sum_{i=1}^{\infty} \frac{i^{n}}{n !} U_{m}(n ; t) x^{n}
$$

Fourier transforms of the characteristic functions $G(x ; t)$ and $Q(x ; t)(2.13)$ define the probability measures of the value $v(C ; t)$ and the volume $\mu(U ; t)$ (2.6) during the interval $\Delta$ (1.4) similar to (2.10): 


$$
v(C ; t)=\int d x G(x ; t) \exp (-i x C) ; \quad \mu(U ; t)=\int d x Q(x ; t) \exp (-i x U)
$$

The statistical moments of the value $C_{m}(n ; t)$ and the volume $U_{m}(n ; t)(2.5)$ take form:

$$
\begin{aligned}
& C_{m}(n ; t)=\left.\frac{d^{n}}{(i)^{n} d x^{n}} G(x ; t)\right|_{x=0}=\int d C C^{n} v(C ; t) \\
& U_{m}(n ; t)=\left.\frac{d^{n}}{(i)^{n} d x^{n}} Q(x ; t)\right|_{x=0}=\int d U U^{n} \mu(U ; t)
\end{aligned}
$$

We take the frequency-based probability measures $v(C ; t)$ and $\mu(U ; t)(2.6)$ of the value $C(t)$ and the volume $U(t)$ as the ground that determines the price statistical moments $p(n ; t)(2.4 ; 2.8)$, the price characteristic function $F(x ; t)$ (2.12) and the price probability measure $\eta(p ; t)(2.10)$. Relations $(2.1-2.16)$ state a very simple and economically justified result: the price probability measure $\eta(p ; t)(2.9)$ and the price statistical moments $p(n ; t)$ entirely depend on the probabilities measures of the value $\nu(C ; t)$ and the volume $\mu(U ; t)(2.12-2.16)$. One may consider the relations (2.1-2.16) as a formal mathematical expression of a common statement: "You cannot beat the market". Who want predict the price probability measure $\eta(p ; t)$ must predict the probability measures of the value $\nu(C ; t)$ and the volume $\mu(U ; t)$ and that problem equals full forecast of the entire market. We leave further studies of $(2.1-2.16)$ for future.

The description of the price probability measure $\eta\left(p_{i} ; t\right)(2.2)$ for discrete $p_{i}, i=1, \ldots i(N)$ replaces the integrals in (2.9-2.11) by the sums but that do not change the model essentially. We omit the trivial transition from the case with a continuous probability measure $\eta(p ; t)(2.9-2.11)$ to the case with a discrete probability measure $\eta\left(p_{i} ; t\right)$. We remind that the first two price statistical moments $p(1 ; t)$ and $p(2 ; t)(2.4-2.8)$ define the price volatility $\sigma_{p}{ }^{2}(t)$ with respect to the price probability measure $\eta(p ; t)(2.2)$. Indeed, the price volatility ${\sigma_{p}}_{p}^{2}(t)$ equals the difference between the mean square price $p(2 ; t)$ and square of the mean price $p^{2}(1 ; t)$ :

$$
\sigma_{p}^{2}(t)=p(2 ; t)-p^{2}(1, t)=\frac{C(2 ; t)}{U(2 ; t)}-\frac{C^{2}(1 ; t)}{U^{2}(1 ; t)}
$$

Relations (2.17) present the price volatility ${\sigma_{p}}^{2}(t)$ with respect to the probability measure $\eta(p ; t)$ as a function of $C(n ; t)$ and $U(n ; t)(2.7)$ for $n=1,2$. To forecast the price volatility ${\sigma_{p}}^{2}(t)(2.17)$ one should model the dynamics of $p(1 ; t)$ and $p(2 ; t)(2.4-2.8)$. Description of the mean price $p(1 ; t)$ is determined by evolution of the sums of the value $C(1 ; t)$ and the volume $U(1 ; t)$ of trades during $\Delta$. The variables $C(1 ; t)$ and $U(1 ; t)$ are determined by the sums of the market trades of the first degree and we call their description as the first-order economic theory (Olkhov, 2018-2019b).

However, prediction of the price volatility $\sigma_{p}^{2}(t)(2.17)$ and $p(2 ; t)$ requires modeling the sums of squares of the values $C(2 ; t)$ and the volumes $U(2 ; t)$ during $\Delta$. The direct dependence of the price volatility $\sigma_{p}^{2}(t)$ on $p(2 ; t)$ and $C(2 ; t)$ and $U(2 ; t)$ uncovers hidden complexity of the price volatility predictions. To forecast the price volatility $\sigma_{p}^{2}(t)$ one should describe $C(1 ; t)$ and $U(1 ; t)$ and also model $C(2 ; t)$ and $U(2 ; t)(2.7)$. Current macroeconomic theories do not consider the variables determined by the sums of squares of the value and the volume of trades or the sums of 
squares of agents' investment, consumption, etc. To describe these macro variables one should develop a new secondorder economic theory.

Indeed, current macroeconomic and financial models describe the economic and financial variables of the firstdegree. For example, the macro investment is determined as the investment made by all agents in the economy. The macro credit, taxes, consumption, etc., are determined as sums (without duplication) of the credits, taxes, consumption, etc., of all agents in the economy during $\Delta$. All these macroeconomic variables are composed as sums of the first-degree variables. The forecasts of the price volatility $\sigma_{p}^{2}(t)(2.17)$ requires the modeling of the sums of squares of the value and the volume of market trades. It is obvious that the second-degree trades $C(2 ; t)$ and $U(2 ; t)$ (2.7) interact with the second-degree economic and financial variables involved into transactions. In particular, $C(2 ; t)$ impacts sums of squares of agents' funds involved into transactions, $U(2 ; t)$ impacts sums of squares of agents' commodities involved into transactions. The description of $C(2 ; t)$ and $U(2 ; t)(2.7)$ establishes the ground for modeling the second-degree macroeconomic variables and their volatilities. It is a tough problem and in the next section we only start study the second-degree economic theory.

Brief results. We introduce the new price probability measure $\eta(p ; t)$ through the price characteristic function, determined by the set of price statistical moments. Each nth statistical moment $p(n ; t)$ of the price equals the ratio $C_{m}(n ; t) / U_{m}(n ; t)$ of the value statistical moment $C_{m}(n ; t)$ to the volume statistical moment $U_{m}(n ; t)$ of all trades performed during an averaging interval $\Delta$. The price probability measure $\eta(p ; t)$ entirely depends on the probability measures $\nu(C ; t)$ and $\mu(U ; t)$ of the value and the volume.. The relations (2.1-2.16) give formal mathematical expression of a common statement: "You cannot beat the market". One who wants predict the price probability measure $\eta(p ; t)$ must predict the probability measures of the market value $\nu(C ; t)$ and the volume $\mu(U ; t)$.

\section{Sums of squares of the value and the volume}

In this section we describe the sums of squares of the value and the volume $C(2 ; t)$ and $U(2 ; t)(2.6)$ of the market trades using methods developed in (Olkhov, 2016a-2020).

First, we assume that it is possible to develop the unified methodology for the agents' risk assessments based on a numerical continuous risk scoring. Up now letters denote the risk ratings. Each agency - S\&P, (2014, 2016), Moody's, (2010, 2018), Fitch, (2018) - introduces its own letter-ratings methodology. However, at least eighty years ago Durand (1941) and then sixty years ago Myers and Forgy (1963) suggested the numerical credit scoring. Last years researchers use the numerical risk rating to compare the risk assessment provided by the different rating agencies (Beaver et.al., 2006; Poon and Shen, 2020). We assume that no internal econometric obstacles may prevent the transfer from letters to a numerical risk scoring. This transition depends mostly on the business interests of the major ratings companies. The development of a unified methodology that can be adopted by the major business players should deliver benefits to the rating agencies, investors, financial markets, business and economic authorities. The unified numerical continuous risk grades methodology will significantly improve the methods for the modeling macroeconomic and financial processes and management of economic policy. Introduction of the continuous risk grades is a natural development of the numerical grades methodology. We do not specify any particular risks like the 
credit or the inflation risk but consider all possible economic, financial, political, etc., risks those impact the financial processes.

Second, we assume that it is possible to assess the risk ratings for the almost all economic agents like international banks, corporations, local firms, companies and even households. That will distribute all economic agents by their numerical risk ratings as coordinates of the risk space.

Third, the agents in the economy always act under the pressure of numerous risks. The assessments of the agents' ratings for one risk distribute the agents in a 1 -dimensional risk space. Simultaneous assessments of the agent's ratings for two or three risks distribute the agents in a two or three dimensional risk space. Absolute numerical values of the risk ratings have no sense. For certainty, we assume that for any risk the most secure rating should be equal zero and the most risky rating equals 1 . Thus the agents with the continuous numerical ratings of a single risk fill a unit interval $[0,1]$ and the agents with the continuous numerical ratings of $m$ risks fill a unit cube in space $R^{m}$.

Forth. The explicit description of numerous separate agents of the entire economy is almost impossible. It requires too much exact data of the economic and financial variables for millions of separate agents, the data of all market trades between the agents, the data of the agent's expectations, the risk assessments of all agents, etc. It is almost impossible to imagine that one can collect the precise data for all agents. Actually, numerous small perturbations would move the forecasts in the wrong directions. To solve the problem we reduce the accuracy of the economic description. We aggregate and smooth out the description of the agents in the risk economic space and introduce the continuous economic media approximation (Olkhov, 2016a, 2018; 2019a -2019c) that describes the collective agents' variables, trades and expectations. Below we explain the meaning of the continuous media approximation for the market trades in more detail.

The benefits of our approach for the economic modeling are follows. First, the transition from the letters to the continuous numerical risk scoring uncovers the hidden motion of the economic agents. Indeed, the rating agencies for decades use the transition matrix to describe the probability of the agent's transfer from a particular risk grade to a different one (Belkin, 1998; Schuermann and Jafry, 2003; Ho et.al, 2017; S\&P, 2018). Introduction of the numerical continuous scoring defines the numerical distance between the risk ratings and hence, introduces the mean velocity of the agent's risk transition. The motion of the agents in the risk economic space during the transition from one risk rating to another defines the flows of macro variables, trades and expectations carried by the economic agents within the risk motion. We use this approach to describe $C(2 ; t)$ and $U(2 ; t)$ and refer (Olkhov, 2018, 2019b, 2019c) for all further definitions and detail.

\section{Second-degree trades}

To start with we consider the economy as a system of numerous economic agents those perform the economic and financial transactions. We assume that the agents perform the market trades under the action of $m$ risks and it is possible to assess the risk ratings for all agents in the economy. Let us take that each agent $i$ is determined by its risk coordinates - the numerical continuous risk ratings $\boldsymbol{x}$

$$
\boldsymbol{x}=\left(x_{1}, \ldots x_{m}\right) ; 0 \leq x_{i} \leq 1 ; i=1, . . m
$$


We consider the risk assessments of the agents as the procedures similar to the measurements of coordinates of physical particles and call the agents' numerical continuous risk ratings $\boldsymbol{x}$ (3.1) as the agents' coordinates in the economic domain (3.2) of the risk space $R^{m}$ :

$$
0 \leq x_{i} \leq 1 ; i=1, . . m
$$

Let us take that an agent $i$ with risk coordinates $\boldsymbol{x}$ executes a transaction with an agent $j$ with risk coordinates $\boldsymbol{y}$. To simplify the model we assume that at a moment $t$ the agent $i$ at a point $\boldsymbol{x}$ sells the volume $U_{i j}$ of the variable $E$ to the agent $j$ at a point $\boldsymbol{y}$. One may consider as the variable $E$ the assets, commodities, goods, currency, service, credits, investment etc. Assume that the trade volume $U_{i j}$ of this particular transaction between the agents $i$ and $j$ has the trade value $C_{i j}$. We define the market trade of the first-degree at a moment $t$ between the agents $i$ and $j$ as a two component function $\boldsymbol{b}_{i j}$ :

$$
\boldsymbol{b}_{i j}(1 ; t, \mathbf{z})=\left(U_{i j}(t, \mathbf{z}) ; C_{i j}(t, \mathbf{z})\right) ; \boldsymbol{z}=(\boldsymbol{x}, \boldsymbol{y})
$$

The coordinates $\boldsymbol{x}$ and $\boldsymbol{y}$ of the agents $i$ and $j$ involved into this trade establish the economic domain with double dimension $(3.1 ; 3.2 ; 3.4 ; 3.5)$ with coordinates $z=(\boldsymbol{x}, \boldsymbol{y})$ :

$$
\begin{aligned}
& \boldsymbol{z}=(\boldsymbol{x}, \boldsymbol{y}) ; \boldsymbol{x}=\left(x_{1} \ldots x_{m}\right) ; \boldsymbol{y}=\left(y_{1} \ldots y_{m}\right) \\
& 0 \leq x_{i} \leq 1 ; 0 \leq y_{j} \leq 1 ; i=1, \ldots m ; j=1, \ldots m
\end{aligned}
$$

We define the price $p_{i j}$ of the trade $\boldsymbol{b}_{i j}$ (3.3) between the agents $i$ and $j$ similar to (2.1):

$$
C_{i j}(t, \mathbf{z})=p_{i j}(t, \mathbf{z}) U_{i j}(t, \mathbf{z})
$$

Now we define the second-degree trade $\boldsymbol{b}_{i j}(2 ; t, z)$ as:

$$
\boldsymbol{b}_{i j}(2 ; t, \mathbf{z})=\left(U_{i j}^{2}(t, \mathbf{z}) ; C_{i j}^{2}(t, \mathbf{z})\right)
$$

We define the transactions $\boldsymbol{b}_{i j}(n ; t, z)$ of degree $n$ as

$$
\boldsymbol{b}_{i j}(n ; t, \mathbf{z})=\left(U_{i j}^{n}(t, \mathbf{z}) ; C_{i j}^{n}(t, \mathbf{z})\right)
$$

We determine the price of $n t h$ degree $p^{n}{ }_{i j}(t, z)$ of the trade $\boldsymbol{b}_{i j}(n ; t, z)(3.8)$ similar to (2.3) as:

$$
\begin{aligned}
& C_{i j}^{n}(t, \mathbf{z})=p_{i j}^{n}(t, \mathbf{z}) U_{i j}^{n}(t, \mathbf{z}) \\
& C_{i j}^{n}(t, \mathbf{z})=p_{i j}^{n}(t, \mathbf{z}) U_{i j}^{n}(t, \mathbf{z})
\end{aligned}
$$

To avoid the excessive accuracy related with the description of the trades between numerous separate agents we introduce the economic continuous media approximation for the second-degree trades. We replace description of the second-degree trades $\boldsymbol{b}_{i j}(2 ; t, z)$ between the individual agents $i$ at $\boldsymbol{x}$ and $j$ at $\boldsymbol{y}$ by description of the collective second- 
degree trades $\boldsymbol{B}(2 ; t, \boldsymbol{z}), \boldsymbol{z}=(\boldsymbol{x}, \boldsymbol{y})$ between the points $\boldsymbol{x}$ and $\boldsymbol{y}$. To do that we introduce a small scale $d$ and a unit volume $d V(z)$ in the economic domain $(3.4,3.5)$

$$
d V(\mathbf{z})=d V(\boldsymbol{x}) d V(\boldsymbol{y}) ; d V(\boldsymbol{x})=d^{n} ; d V(\boldsymbol{y})=d^{n} ; \boldsymbol{z}=(\boldsymbol{x}, \boldsymbol{y})
$$

We assume that the scale $d \ll<1$ but each unit volume $d V(\boldsymbol{x})$ and $d V(\boldsymbol{y})$ contains a lot of agents with the risk coordinates inside $d V(\boldsymbol{x})$ and $d V(\boldsymbol{y})$. Let us assume that during $\Delta$ the agents inside $d V(\boldsymbol{x})$ and $d V(\boldsymbol{y})$ perform a lot of mutual trades. We define the collective second-degree trade $\boldsymbol{B}(2 ; t, z)$ between points $\boldsymbol{x}$ and $\boldsymbol{y}$ as a sum of all seconddegree trades $\boldsymbol{b}_{i j}(2 ; t, \boldsymbol{z})$ of the agents $i$ with coordinates in a unit volume $d V(\boldsymbol{x})$ and the agents $j$ with coordinates in a unit volume $d V(y)$ (3.10) and then average this sum during $\Delta$ (A1-A.5). To simplify the reading we move most calculations into the Appendices. The second-degree collective trades $\boldsymbol{B}(2 ; t, \boldsymbol{z})$ (A.1-A.5) define the second-degree price $p(2 ; t, z)$ of the trades between the agents at point $\boldsymbol{x}$ and the agents at point $\boldsymbol{y}$ in the domain $(3.4,3.5)$ similar to (2.6):

$$
C(2 ; t, \mathbf{z})=p(2 ; t, \mathbf{z}) U(2 ; t, \mathbf{z})
$$

(3.11) describes the mean second-degree price $p(2 ; t, \boldsymbol{z})$ between points $\boldsymbol{x}$ and $\boldsymbol{y}$ averaged during $\Delta$. The relation $(2.6 ; 2.7)$ for $n=2$ describe the mean square of the price $p(2 ; t)$ of all trades in the economy averaged during $\Delta$. To derive $(2.6 ; 2.7)$ using the definitions of $\boldsymbol{B}(2 ; t, z)$ (A.1-A.5) let us take integral by the collective second-degree trades over the economic domain $(3.4,3.5)$ or calculate the sum over all agents in the economy involved into the trades during $\Delta$ :

$$
\begin{gathered}
\boldsymbol{B}(2 ; t)=(U(2 ; t) ; C(2 ; t)) \\
U(2 ; t)=\int d \mathbf{z} U(2 ; t, \mathbf{z}) ; C(2 ; t)=\int d \mathbf{z} C(2 ; t, \mathbf{z}) \\
C(2 ; t)=p(2 ; t) U(2 ; t)
\end{gathered}
$$

The relations (3.14) define the price $p(2 ; t)$ similar to (2.6) for $n=2$. Thus, to describe the price $(2.6 ; 2.7)$ one should describe the second-degree trades $\boldsymbol{B}(2 ; t)$ and $\boldsymbol{B}(2 ; t, \boldsymbol{z})$.

\section{Flows of the second-degree trades}

In this subsection we introduce the notions of the trade flows in the economic domain $(3.4,3.5)$. The rating agencies for decades provide the assessments of the risk transition matrices (Belkin, 1998; Schuermann and Jafry, 2003; Ho et.al, 2017; S\&P, 2018). Elements $a_{i j}$ of the transition matrix define the probabilities of the agents' transition from the risk grade $i$ to the risk grade $j$ during certain time interval $T$. As usual the interval $T$ equals a half a year, one, two three years. If one uses the numerical continuous risk grades then the transition matrices get completely different and much more important and reasonable economic meaning. For the numerical continuous risk grades the transition from rating $\boldsymbol{x}_{i}$ to $\boldsymbol{x}_{j}$ defines the numerical risk interval $\boldsymbol{l}_{i j}$ : 


$$
\boldsymbol{l}_{i j}=x_{j}-\boldsymbol{x}_{i}
$$

Let us take that the transition from $\boldsymbol{x}_{i}$ to $\boldsymbol{x}_{j}$ takes time T. Hence, the element $a_{i j}$ of the transition matrix defines the probability of the agent's motion in the economic domain (3.2) during time $T$ with the velocity $\boldsymbol{v}_{i j}$ :

$$
\boldsymbol{v}_{i j}=\frac{\boldsymbol{l}_{i j}}{T} \text { with probability } a_{i j} ; \quad \sum_{j} a_{i j}=1
$$

Hence, the transition matrices can define the mean velocity $\boldsymbol{v}_{i}$ of the agent at point $\boldsymbol{x}_{i}$ during the time interval $T$ as:

$$
\boldsymbol{v}\left(t, x_{i}\right)=\sum_{j=1}^{K} \boldsymbol{v}_{i j} a_{i j}=\frac{1}{T} \sum_{j=1}^{K} \boldsymbol{l}_{i j} a_{i j}
$$

Here $K$ means the number of the different numerical risk grades that defines the degree $K \mathrm{x} K$ of the transition matrix. Thus the transition matrices can define the mean velocities of the agents in the economic domain. The risk motion of the agents with the velocity (3.17) induces the flows of the economic and financial variables carried by the agents in the risk domain and the flows of market trades as well (Olkhov, 2018-2019c). Indeed, each agent in the economic domain with the velocity $v$ (3.17) carries its economic and financial variables. Collective effect of such transport of the economic variables by all agents in a small volume $d V(\boldsymbol{x})$ is described by the flows of the variables in (3.2). The same time the motion of the agents with velocity (3.17) defines the flows (A.6-A.19) of collective trades in the economic domain with double dimension $(3.4 ; 3.5)$.

The flows $\boldsymbol{P}(2 ; t, \boldsymbol{z})$ (A.10-A.17) of the second-degree transaction $\boldsymbol{B}(2 ; t, \boldsymbol{z})$ between the points $\boldsymbol{x}$ and $\boldsymbol{y}$ describe the amounts of the second-degree volume $U(2 ; t, z)(\mathrm{A} .4)$ and the value $C(2 ; t, \boldsymbol{z})(\mathrm{A} .5)$ of the trades $\boldsymbol{B}(2 ; t, \boldsymbol{z})$ (A.1) carried by the transactions' velocities $v(2 ; t, z)$ (A.17; A.18) through the $2 m$-dimensional economic domain $(3.4,3.5)$. The velocity $\boldsymbol{v}_{U}(2 ; t, z)$ (A.19) defines the motion of the second-degree volume $U(2 ; t, z)$ (A.4) and may be different from the velocity $v_{C}(2 ; t, z)$ (A.19) that describes the motion of second-degree value $C(2 ; t, z)(\mathrm{A} .5)$.

The second-degree trades help describe the different forms of the price and in App. A we introduce the average square price $p_{s}(2 ; t, \boldsymbol{x})$ of all sell-trades from the point $\boldsymbol{x}$ and the average square price $p_{b}(2 ; t, \boldsymbol{y})$ of all buy-trades at the point $\boldsymbol{y}$ at moment $t$ averaged during $\Delta$ as:

$$
\begin{aligned}
& C_{s}(2 ; t, \boldsymbol{x})=p_{s}(2 ; t, \boldsymbol{x}) U_{s}(2 ; t, \boldsymbol{x}) \\
& C_{b}(2 ; t, \boldsymbol{y})=p_{b}(2 ; t, \boldsymbol{y}) U_{b}(2 ; t, \boldsymbol{y})
\end{aligned}
$$

Brief results. Evolution of the sums of squares of the value and the volume of the trades depends on the trade flows. Prediction of the price volatility requires description of squares of the market trades and their flows and should take into account impact of the agent's expectations.

\section{Collective expectations}

In this Sec. we introduce the new economic notions - the collective expectations and the collective expected trades. The collective expectations approve execution of the second-degree trades (3.12-3.14). We use the collective expectations to derive equations that describe small disturbances of the price volatility. 
It is generally considered that the agents take trade decisions under their expectations. The expectations play the crucial role for the market trade performance. Decades of the research describe the impact of the expectations on financial markets, price dynamics and economic evolution (Muth, 1961; Lucas, 1972; Blume and Easley, 1984; Brunnermeier and Parker, 2005; Dominitz and Greenwood and Shleifer, 2014). Observations and measurements of the expectations is a difficult problem (Manski, 2004; Dominitz and Manski, 2005; Janžek and Ziherl, 2013; Manski, 2017). A lot of efforts are spent to observe, identify, estimate and measure the expectations. These problems are very complex. We suggest simplify the description of the expectations and their impact on the performance of market trades. To do that we regard the expectations as the agents' assumptions and forecasts of the numerous economic and financial variables, the price and the inflation trends, the market trade activity, the expectations of other agents, the forecasts of any factors that can impact the economy, etc. We assume that the agents execute the trades under the action of multiple expectations. Each agent may have multiple expectations and can perform similar trades under the action of different expectations. Below we model the action of multiple expectations on the market trades.

It seems that our assumption on multiplicity of the expectations increases the complexity of the model: the difficulties of identification and measurement of the expectations are increased by the huge amount of different expectations of each agent. To overcome that, we aggregate the expectations of different agents alike to aggregation of the trades (A.1-A.5) performed by the agents with risk coordinates inside the unit volume $d V(z)(3.10)$ in the economic domain $(3.4,3.5)$. We roughen the description of the expectations of separate agents and introduce the aggregated smooth collective expectations. This approach is similar to the description of the macroeconomic variables and transactions developed in (Olkhov, 2016a-2019c) via the continuous economic media approximation.

Multiplicity of the expectations arises the problem of their comparative effect on the market. The agent's expectations differ from the agent's economic and financial variables. The agents' variables like the investment, the assets, the consumption - are additive. The sum of the assets of a group of agents (without duplication) defines the assets of the group of agents. But the expectations are not additive. Sum of the expectations have no economic sense. The economic impact of the expectation that approve $\$ 1$ bln deal must be a little more valuable than the impact of the expectation that approve $\$ 1$ deal. To collect the agents' expectations those approve the second-degree trades (3.7) one must take into account the economic value of the trades approved by these expectations. One cannot simply sum the agents' expectations to derive the collective expectations of numerous agents. We state that the market value of the collective expectation with respect to the definite second-degree trade should be proportional to the market value and the market volume of that second-degree trade (3.7) performed under the selected expectations. We assume that the agents take decisions on squares of the volume $U^{2}$ and the value $C^{2}$ of the trade under different expectations. Thus, the expectation that approves $U^{2}$ of the second-degree trade (3.7) should be weighted by the square of the volume $U^{2}$. The expectation that approves the square of the value $C^{2}$ should be weighted by $C^{2}$. We refer to (Olkhov, 2019b; 2019c) for details and apply that method for the description of the collective expectations of the second-degree trades $\boldsymbol{b}_{i j}(2 ; t, \boldsymbol{z})$ (3.7). Now we describe the collective expectations in a more formal manner.

Assume that the agents in the economy have $j=1, \ldots K$ different expectations. Each agent $i$ that is involved into a transaction $\boldsymbol{b}_{i j}(2 ; t, \boldsymbol{z})$ (3.7) with an agent $j$ should take a decision on the volume $U_{i j}$ and the value $C_{i j}$ of the trade. Thus, any trade is performed under the action of four expectations - two expectations of the agent $i$ and two expectations 
of the agent $j$ approve the volume $U_{i j}$ and the value $C_{i j}$ of the trade. Propose that the agent $i$ takes a decision on the volume $U_{i j}$ under the expectation $e x_{U i}(k ; t, \boldsymbol{x})$ of type $k=1, . . K$, and a decision on the value $C_{i j}$ under the expectation $e x_{C i}(l ; t, \boldsymbol{x})$ of type $l=1, . . K$. We propose that the expectations $e x_{U i}(k ; t, \boldsymbol{x})$ those approve the volumes $U_{i j}(k ; t, z)$ of trades may depend on the expectations $e_{C i}(l ; t, \boldsymbol{x})$ those approve the values $C_{i j}(l ; t, z)$ and vice versa. Hence, the volumes $U_{i j}$ and values $C_{i j}$ of the trades performed by the seller $i$ can depend on both expectations $k$ and $l$ : ex $x_{U i}(k, l ; t, \boldsymbol{x})$ and $\operatorname{ex}_{C i}(k, l ; t, \boldsymbol{x})$. We denote the second-degree trade $\boldsymbol{b}_{i j}(2 ; \boldsymbol{k} ; t, \boldsymbol{z})$ as the pair $\left(U_{i j}{ }^{2}, C_{i j}{ }^{2}\right)$ of the squares of the volume and the value of the trade performed under the expectations of the type $k=(k, l)$ of the seller $i$ at the point $\boldsymbol{x}$ with the buyer $j$ at the point $y$ as:

$$
\boldsymbol{b} \boldsymbol{s}_{i j}(2 ; \boldsymbol{k} ; t, \boldsymbol{z})=\left(U_{i j}^{2}(\boldsymbol{k} ; t, \mathbf{z}) ; C_{i j}^{2}(\boldsymbol{k} ; t, \mathbf{z})\right) ; \boldsymbol{k}=(k, l) ; k, l=1, \ldots K ; \boldsymbol{z}=(\boldsymbol{x}, \boldsymbol{y})
$$

Let us denote $e x_{U i}(\boldsymbol{k} ; t, \boldsymbol{x})$ as the expectations of the type $\boldsymbol{k}=(k, l)$ of the seller $i$ at the point $\boldsymbol{x}$ that determine the decision to perform the trade with the volume $U_{i j}$. Let us denote $\operatorname{ex}_{C i}(\boldsymbol{k} ; t, \boldsymbol{x})$ as the expectations of type $\boldsymbol{k}=(k, l)$ of the seller $i$ at the point $\boldsymbol{x}$ that determine the decision to perform the trade with the value $C_{i j}$. Let us denote the pair $\boldsymbol{e x}(\boldsymbol{k} ; t, \boldsymbol{x})$ (4.2) as the expectations of the seller $i$ those approve the trade (3.3).

$$
\boldsymbol{e x}(\boldsymbol{k} ; t, \boldsymbol{x})=\left(e x_{U i}(\boldsymbol{k} ; t, \boldsymbol{x}) ; \operatorname{ex}_{C i}(\boldsymbol{k} ; t, \boldsymbol{x})\right)
$$

We use different notations $(3.7 ; 3.8 ; 4.1)$ of the same trade $(3.3)$ to outline that description of the sums of trades (3.8) for each $n=1,2$.. and description of the expectations weighted by $n t h$ power of the value and the volume are different and depend on $n$.

We propose that all expectations $\boldsymbol{e x}(\boldsymbol{k} ; t, \boldsymbol{x})$ have the same measure and a sum of two expectations has meaning of an expectation. Thus we propose that one can sum different expectations and derive the sum of the expectations weighted by the volume or the value. The relations $(4.1,4.2)$ define the second-degree trades and the expectations of a seller $i$ at a point $\boldsymbol{x}$. Similar relations define the second-degree trades $\boldsymbol{b}_{i j}(2 ; t, z ; \boldsymbol{l})$ and the expectations $\boldsymbol{e x}(t, \boldsymbol{y} ; \boldsymbol{l})$ of a buyer $j$ at a point $\boldsymbol{y}$.

$$
\boldsymbol{b} \boldsymbol{s}_{i j}(2 ; t, \mathbf{z} ; \boldsymbol{l})=\left(U_{i j}^{2}(t, \mathbf{z} ; \boldsymbol{l}) ; C_{i j}^{2}(t, \mathbf{z} ; \boldsymbol{l})\right) ; \boldsymbol{l}=(k, l) ; k, l=1, \ldots K ; \boldsymbol{z}=(\boldsymbol{x}, \boldsymbol{y})
$$

The buyers' expectations $\boldsymbol{e x}(t, \boldsymbol{y} ; \boldsymbol{l})$ approve trades (4.2) of the agent $j$ at the point $\boldsymbol{y}$ :

$$
\boldsymbol{e x}(t, \boldsymbol{y} ; \boldsymbol{l})=\left(e x_{U j}(t, \boldsymbol{y} ; \boldsymbol{l}) ; \boldsymbol{e x} x_{C j}(t, \boldsymbol{y} ; \boldsymbol{l})\right) ; \boldsymbol{l}=(k, l) ; k, l=1, \ldots K
$$

To describe the collective expectations of the agents with coordinates inside (3.10) we introduce a new additive financial issue - the expected trades. We consider the expected value of trades as a factor determined by the agents' expectations those approve the trade value multiplied by the square of the value. Accordingly we define the expected volume trades as expectations those approve the trade volume multiplied by the square of the volume. We introduce the second-degree sellers' expected trades $\boldsymbol{e t}_{i j}(\boldsymbol{k} ; t, \boldsymbol{z})$ as the sellers' $\boldsymbol{e x}(\boldsymbol{k} ; t, \boldsymbol{x})$ expectations (4.2) multiplied by squares of the volumes $U^{2}{ }_{i j}$ and squares of the values $C^{2}{ }_{i j}$ of trades: 


$$
\begin{array}{r}
\boldsymbol{e t}_{i j}(\boldsymbol{k} ; t, \mathbf{z})=\left(e t_{U i j}(\boldsymbol{k} ; t, \mathbf{z}) ; e t_{C i j}(\boldsymbol{k} ; t, \mathbf{z})\right) \\
e t_{U i j}(\boldsymbol{k} ; t, \mathbf{z})=e x_{U i}(\boldsymbol{k} ; t, \boldsymbol{x}) U_{i j}^{2}(\boldsymbol{k} ; t, \mathbf{z}) \\
e t_{C i j}(\boldsymbol{k} ; t, \mathbf{z})=e x_{C j}(\boldsymbol{k} ; t, \boldsymbol{y}) C_{i j}^{2}(\boldsymbol{k} ; t, \mathbf{z})
\end{array}
$$

The relations similar to (4.5-4.7) define the second-degree buyers' expected trades $\boldsymbol{e t}_{i j}(t, z ; \boldsymbol{l})$. We use the procedure similar to (A.1-A.5) and (A.10-A.19) to define the sums of the second-degree expected trades and their flows (see Appendix B). We introduce the collective sellers' expectations $E x(\boldsymbol{k} ; t, \boldsymbol{z})($ B.12-B14) and the collective buyers' expectations $E x(t, z ; l)$ as the average expectations weighted by the sums of squares of the value and the volume (B.15B.17). The integral by the economic domain $(3.4,3.5)$ introduces the collective sellers' $\operatorname{Ex}(\boldsymbol{k} ; t)($ B.20, B.210) and buyers' $E x(t ; \boldsymbol{l})$ (B.22, B.23) expectations as a function of time $t$ and types $\boldsymbol{k}$ or $\boldsymbol{l}$ of expectation $(4.3,4.4)$. Below we use these expectations to model oscillations of the price $p(2 ; t)(2.4 ; 2.7)$.

Brief results. In this Section we introduce notions of the collective expected trades as the collective expectations weighted by the squares of the value and the volume. In App.C we derive the equations on the collective seconddegree trades and the collective expectations in a closed form and describe the price volatility perturbations.

\section{Mean price and price volatility perturbations}

In this Section in a linear approximation by trade perturbations we describe the mean price and the price volatility perturbations through the disturbances of sums of the first and the second-degree values and volumes. Most calculations and equations are presented in App. $C$ and D.

In App. C we derive the system of equations on the second-degree trades $\boldsymbol{B}(2 ; t, z)$ (A.1-A.5) and their flows $\boldsymbol{P}(2 ; t, z)$ (A.11-A.19) and on the expected trades according to (Olkhov, 2019b). The system of equation (C.1-C.5) and the aggregated form of equations (C.6-C.9) those describe the sums of the squares of the value and the volume of the entire economy as the functions of the expectations of type $\boldsymbol{k}$ and time $t$ only - are complex. We present the simple consequences of equations (C.5-C.9) that describe the mean price and the price volatility fluctuations.

In Olkhov (2019b) we show how equations on the first-degree trades imply equations on the mean price $p(1, t)$ (1.3). Here we derive the similar equations on the mean square price $p(2, t)(2.4 ; 2.7)$ for $n=2$. Let us neglect the action of the flows in (C.6, C.7) and take the equations on the second-degree trades as:

$$
\frac{d}{d t} U(2, \boldsymbol{k} ; t)=F_{U}(\boldsymbol{k} ; t) ; \frac{d}{d t} C(2, \boldsymbol{k} ; t)=F_{C}(\boldsymbol{k} ; t)
$$

Let us remind the form of the second-degree price $p(2, \boldsymbol{k} ; t)$ as

$$
C(2, \boldsymbol{k} ; t)=p(2, \boldsymbol{k} ; t) U(2, \boldsymbol{k} ; t)
$$

Equations $(5.1,5.2)$ on $U(2 ; \boldsymbol{k}, t)$ and $C(2 ; \boldsymbol{k}, t)$ allow present equations on $p(2 ; \boldsymbol{k}, t)$ as:

$$
U(2, \boldsymbol{k} ; t) \frac{d}{d t} p(2, \boldsymbol{k} ; t)+p(2, \boldsymbol{k} ; t) F_{U}(\boldsymbol{k} ; t)=F_{C}(\boldsymbol{k} ; t)
$$


Equations $(5.1,5.3)$ show that the dynamics of the price $p(2, \boldsymbol{k} ; t)$ or similar equations $(5.4)$ for the price $p(2 ; t)$ and the volume $U(2 ; t)(3.13,3.14)$ :

$$
\frac{d}{d t} U(2 ; t)=F_{U}(t) ; U(2 ; t) \frac{d}{d t} p(2 ; t)+p(2 ; t) F_{U}(t)=F_{C}(t)
$$

are determined by functions $F_{U}, F_{C}$. As we discussed above, $F_{U}, F_{C}$ can depend on the expectations, the expected transactions and their flows. Even if one neglects the impact of the trades' flows $P_{U}, P_{C}$ (A.25-A.28) and the flows of the expected transactions (B.28, B.29) and equations (C.3, C.5), equations $(5.3,5.4)$ on the price $p(2, k ; t)$ or the price $p(2 ; t)$ depend on functions $F_{U}, F_{C}$ that model dynamics of the second-degree trades. Equations on $p(2, \boldsymbol{k} ; t)$ or $p(2 ; t)$ and similar equations on $p(1 ; t)\left(\right.$ Olkhov, 2019) describe evolution of the price volatility $\sigma_{p}^{2}(2.17)$ determined by the first and the second-degree trades.

Equations (5.1) describe fluctuations of the price $p(2, \boldsymbol{k} ; t)$. To show that let us assume that in a linear approximation by perturbations the functions $F_{U}$ and $F_{C}$ in (5.1) depend on the perturbations of the collective expected trades $E t_{U}(\boldsymbol{k} ; t)$ and $E t_{C}(\boldsymbol{k} ; t)\left(\right.$ B.19) as (D.9). Let us take equations on the expected trades $E t_{U}(\boldsymbol{k} ; t)$ and $E t_{C}(\boldsymbol{k} ; t)$ as (C.8) :

$$
\frac{d}{d t} E t_{U}(\boldsymbol{k} ; t)=W_{U}(\boldsymbol{k} ; t) ; \quad \frac{d}{d t} E t_{C}(\boldsymbol{k} ; t)=W_{C}(\boldsymbol{k} ; t)
$$

We assume that in a linear approximation by perturbations the functions $W_{U}$ and $W_{C}$ in (5.5) depend on perturbations of the volume $U(2, \boldsymbol{k} ; t)$ and the value $C(2, \boldsymbol{k} ; t)$ as (D.10). The assumptions $(5.1,5.5)$ describe small oscillations of the collective trades $U(2, k ; t)$ and $C(2, k ; t)$ and hence, describe the fluctuations of the mean square of price $p(2, \boldsymbol{k} ; t)(5.2)$. We describe these fluctuations in Appendix D.

The relations (D.4) express disturbances of the mean square price $p(2 ; t)(3.14)$ though the perturbations of the squares of the volumes $u(2, \boldsymbol{k} ; t)$ (D.1) and the squares of the values $c(2, \boldsymbol{k} ; t)$ (D.1). The relations (D.7, D.8) describe dependence of $p(2 ; t)(3.14)$ on the partial price disturbances $\pi(2, \boldsymbol{k} ; t)$ (D.6) and the volume disturbances $u(2, \boldsymbol{k} ; t)$ (D.1). The disturbances of $p(2 ; t)(\mathrm{D} .4, \mathrm{D} .8)$ and the disturbances of $p(1 ; t)(\mathrm{D} .26)$ describe perturbations of the price volatility $\sigma_{p}^{2}(t)(2.17)$ in the linear approximation (D.27):

$$
\sigma_{p}^{2}(t)=\sigma_{p 0}^{2}\left\{1+\sum_{k, l=1}^{K} \sigma_{2}\left[\mu_{2 \boldsymbol{k}} c(2, \boldsymbol{k} ; t)-\lambda_{2 k} u(2, \boldsymbol{k} ; t)\right]-\sigma_{1}\left[\mu_{1 \boldsymbol{k}} c(1, \boldsymbol{k} ; t)-\lambda_{1 k} u(1, \boldsymbol{k} ; t)\right]\right\}
$$

We underline that the above relations describe disturbances of the price volatility $\sigma_{p}^{2}(t)$ (2.17) obtained by averaging during the time interval $\Delta$. If $\Delta$ equals 1 hour, 1 day or 1 week then during the time terms $T>\Delta$ the price volatility $\sigma_{p}^{2}(t)$ can follow small perturbations, but the frequency of the fluctuations will depend on the averaging interval $\Delta$. We leave further considerations of these problems for future.

Brief results. We describe small perturbations of the mean price, the mean square price and the price volatility at the time scales $T>\Delta$. As we show in App.D, the disturbances of the aggregated trades and the collective expectations may be origin of the exponential growth of the volatility perturbations on the time scales $T>\Delta$. 


\section{Conclusion}

The instantaneous, momentary price time-series have a little usage due to the irregular price oscillations. Investors, traders and researchers aggregate the price time-series during some averaging interval $\Delta$ and obtain the smooth average price, derive the price volatility etc. The choice of the interval $\Delta$ and the choice of the price averaging procedure play important role for the successive investment.

We propose that the conventional price probability measure that is based on the frequencies $n_{p}(1.1)$ of trades at the price $p$ is not the only one and not the best one for the investment decisions and the economic policy. The main defect of the frequency-based price probability (1.1) is its independence from the market trade time-series. It is hard to believe that the statistical properties of the random price may be independent from the market trades' stochasticity. It seems reasonable that the investment decisions and the portfolio strategies should follow the most significant market trades. We introduce the price probability measure $\eta(p ; t)(2.2-2.12)$ that for all $n=1,2, \ldots$ gives the price $n t h$ statistical moment $p(n ; t)$ as $(2.7 ; 2.8)$

$$
C(n ; t)=p(n ; t) U(n ; t)
$$

We define the relations on the price statistical moment $p(n ; t)$ in the form parallel to the relations between the $n t h$ power of the value $C^{n}$, the $n t h$ power of the volume $U^{n}$ and the $n t h$ power of the price $p^{n}$ of the single market trade (2.3). The relations (6.1) imply that the $n t h$ power of the volume $U^{n}$ do not correlate with the $n t h$ power of the price $p^{n}$. However, the $n t h$ power of the volume $U^{n}$ correlates with the $k t h$ power of the price $p^{k}$ for all $k \neq n$. The price volatility $\sigma_{p}^{2}(t)(2.17)$ takes form:

$$
\sigma_{p}^{2}(t)=p(2 ; t)-p^{2}(1, t)=\frac{C(2 ; t)}{U(2 ; t)}-\frac{C^{2}(1 ; t)}{U^{2}(1 ; t)}
$$

To forecast the price volatility ${\sigma_{p}}^{2}(t)(2.17)$ one should predict the sums of the first and the second-degree trades $C(1 ; t), U(1 ; t), C(2 ; t), U(2 ; t)$.

We call the description of the sums of the second-degree values and volumes as the second-order economic theory. We start study the second-order economic theory in the continuous media approximation using the assumptions on the continuous risk grades according to the economic theory frame developed for the description of the first-degree macroeconomic variables, transactions and expectations by Olkhov (2016a-2019c).

The price probability measure $\eta(p ; t)(2.1-2.16)$ entirely depends on the probability measures of the value $\nu(C ; t)$ and the volume $\mu(U ; t)(2.6 ; 2.14)$ of the random market trades performed during the averaging interval $\Delta$. One may consider the relations (2.1-2.16) as the formal mathematical expression of the common statement: "You cannot beat the market". Those who want predict the price probability measure $\eta(p ; t)$ must predict the probability measures of the market trade value $\nu(C ; t)$ and the volume $\mu(U ; t)$ - in other words must derive the full forecast of the entire market. That tough problem unlikely be solved ever and hence, the exact forecast of the price probability measure $\eta$ $(p ; t)$ seems almost unattainable. However, one can develop approximations of the price probability $\eta(p ; t)$. The 
second-order economic theory that models the second-degree trades gives the reasonable forecasts of the price volatility $\sigma_{p}^{2}(t)$, the third-order theory can model the price skewness and so on.

However, investors, traders and researchers are free to choose their own price expectations, price hopes and anticipations and use any price probability measure they prefer. Economics is a social science and in our view no firm rules and regulations of the price averaging methods may exist. The distinctions between the various price definitions and the price probability measures have significant effect on the economic and financial modeling, on the market forecasting and the investment strategies, the portfolio optimization and the hedging models and on sustainability of the economic policy. Only comparisons between the portfolio returns obtained via the investment strategies based on the different price probability measures and procedures can convince investors in the advantages of the price probability measure $\eta(p ; t)$.

We hope that further development of the new price probability measure $\eta(p ; t)$ and the second-order economic theory can bring new and useful financial solutions, improve the investment strategies and the sustainability of the portfolio returns and the economic policy.

\section{References}

Andersen, T., Bollerslev, T., Diebold, F. \& Ebens, H. (2001). The Distribution of Realized Stock Return Volatility. Journal of Financial Economics, 61, 43-76

Andersen, T.G., Bollerslev, T., Christoffersen, P.F. \& Diebold, F.X. (2005). Volatility Forecasting. CFS WP , 2005/08, 1-116

Beaver, W. H., Shakespeare, C. \& Soliman, M.T. (2006). Differential properties in the ratings of certified versus non-certified bond-rating agencies. Journal of Accounting and Economics, 42, 303-334

Belkin, B., Suchower, S. \& Forest, L.R. (1998). A one-parameter representation of credit risk and transition matrices. JP Morgan, CreditMetrics ${ }^{\circledR}$ Monitor, 3-d Q, 46-56

Berkowitz, S.A., Dennis E. Logue, D.E. \& Noser, E.A. Jr. (1988). The Total Cost of Transactions on the NYSE, The Journal of Finance, 43, (1), 97-112

Bernanke, B. \& Gertler, M. (2000). Monetary Policy and Asset Price Volatility. NBER, WP7559, 1-75

Blume, L.E. \& Easley, D. (1984). Rational Expectations Equilibrium: An Alternative Approach. Journal Of Economic Theory, 34, 116-129

Bogousslavsky,V. \& Collin-Dufresne, P. (2019). Liquidity, Volume, and Volatility, Swiss Finance Institute, Research Paper Series $19-69,1-57$

Brock, W.A. \& LeBaron, B.D. (1995). A Dynamic structural model for stock return volatility and trading volume. NBER WP, 4988, 1-46

Brunnermeier, M.K. \& Parker, J.A. (2005). Optimal Expectations. American Economic Review, 95, (4), $1092-1118$

Buryak, A. \& Guo, I. (2014). Effective And Simple VWAP Options Pricing Model, Intern. J. Theor. Applied Finance, 17, (6), 1450036, https://doi.org/10.1142/S0219024914500356

Busseti, E. \& Boyd, S. (2015). Volume Weighted Average Price Optimal Execution, 1-34, arXiv:1509.08503v1

Campbell, J.Y., Grossman, S.J. \& Wang, J. (1993). Trading Volume And Serial Correlation In Stock Returns. The Quarterly Journal of Economics, 905-939

Christiansen, C., Schmeling, M. \& Schrimpf, A. (2012). A Comprehensive Look at Financial Volatility Prediction by Economic Variables. BIS WP, 374, 1-46

CME Group, (2020). $\quad$ www.cmegroup.com/confluence/display/EPICSANDBOX/GovPX+Historical+Data ; www.cmegroup.com/confluence/display/EPICSANDBOX/Standard+and+Poors+500+Futures

Daly, K. (2008). Financial volatility: Issues and measuring techniques, Physica A 387, 2377-2393

Dominitz, J. \& Manski, C.F. (2005). Measuring And Interpreting Expectations Of Equity Returns. NBER, WP 11313, Cambridge, MA

Durand, D. (1941). Volume Title: Risk Elements in Consumer Instalment Financing, Technical Edition, NBER, http://www.nber.org/books/dura41-1

Engle, R.F. \& Patton, A.J. (2001). What good is a volatility model? Quantitative Finance, 1, 237-245

Fama, E.F. (1965). The Behavior of Stock-Market Prices. The Journal of Business, 38, (1), 34-105

Fitch, (2018). Procedures and Methodologies for Determining Credit Ratings. Fitch Ratings, Inc., Form 25-101F1 
Greenwood, R. \& Shleifer, A. (2014). Expectations of Returns and Expected Returns. The Review of Financial Studies, 27 (3), 714-746

Guéant, O. \& Royer, G. (2014). VWAP execution and guaranteed VWAP, SIAM J. Finan. Math., 5(1), 445-471

Hall, R.L. \& Hitch, C.J. (1939). Price Theory and Business Behaviour, Oxford Economic Papers, 2. Reprinted in T. Wilson and P. W. S. Andrews (eds.), Oxford Studies in the Price Mechanism (Oxford, 1951)

Heston, S.L. (1993). A Closed-Form Solution for Options with Stochastic Volatility with Applications to Bond and Currency Options, The Rev. Financial Studies, 6, (2), 327-343

Ho, A., et.al. (2017). Asia-Pacific Structured Finance 2016 Transition and Default Study, FitchRatings Structured Finance, 114

Janžek, T. \& Ziherl, P. (2013). Overview of models and methods for measuring economic agent's expectations. BIS, IFC Bulletin 36, 172-179. https://www.bis.org/ifc/publ/ifcb36.htm

Klyatskin, V.I. (2005). Stochastic Equations through the Eye of the Physicist, Elsevier B.V.

Klyatskin, V.I. (2015). Stochastic Equations: Theory and Applications in Acoustics, Hydrodynamics, Magnetohydrodynamics, and Radiophysics, v.1, 2, Springer, Switzerland

Lucas, R.E. (1972). Expectations and the Neutrality of Money. J. Econ. Theory, 4, 103-124

Mankiw, N.G., Romer, D. \& Shapiro, M.D. (1991). Stock Market Forecastability and Volatility: A Statistical Appraisal, Rev.Economic Studies, 58,455-477

Manski, C. (2004). Measuring Expectations. Econometrica, 72, 1329-1376

Manski, C.F. (2017). Survey Measurement Of Probabilistic Macroeconomic Expectations: Progress and Promise. NBER, WP 23418

Moody's, (2010). Rating Symbols and Definitions. Moody's Investors Service

Moody's, (2018). Procedures and Methodologies Used to Determine Credit Ratings. Moody's Investors Service

Muth, J.F. (1961). Rational Expectations and the Theory of Price Movements, Econometrica, 29, (3) 315-335

Myers, J.H. \& Forgy, E.W. (1963). The Development of Numerical Credit Evaluation Systems, Jour. American Statistical Association, 58, 303, 799-806

Olkhov, V. (2016a). On Economic space Notion. Intern. Rev. Financial Analysis, 47, 372-81.

Olkhov, V. (2016b). Finance, Risk and Economic space. ACRN Oxford Journal of Finance and Risk Perspectives, 5, 209-21

Olkhov, V. (2017a). Quantitative Wave Model of Macro-Finance. Intern. Rev. Financial Analysis, 50, 143-50

Olkhov, V. (2017b). Econophysics of Business Cycles: Aggregate Economic Fluctuations, Mean Risks and Mean Square Risks, 1-31, http://arxiv.org/abs/1709.00282

Olkhov, V. (2018). How Macro Transactions Describe the Evolution and Fluctuation of Financial Variables. Intern. Jour. Financial Studies, 6, 38, 1-19

Olkhov, V. (2019a). Economic Transactions Govern Business Cycles. ACRN Oxford Journal of Finance and Risk Perspectives, 7, 102-22

Olkhov, V. (2019b). Financial Variables, Market Transactions, and Expectations as Functions of Risk. Int. Jour. Financial Stud., $7,66,1-27$

Olkhov, V. (2019c). The Econophysics Of Asset Prices, Returns And Multiple Expectations. The Journal of Network Theory in Finance, 5 (3), 25-52

Olkhov, V. (2020). Volatility depends on Market Trades and Macro Theory. MPRA WP 102434

Padungsaksawasdi, C., \& Daigler, R. T. (2018). Volume weighted volatility: empirical evidence for a new realized volatility measure, Int. J. Banking, Accounting and Finance, 9, (1), 61-87

Pearce, D.K. (1983). Stock Prices and the Economy. FRB Kansas, Economic Review, 7-22

Poon, S-H. \& Granger, C.W.J. (2003). Forecasting Volatility in Financial Markets: A Review, J. of Economic Literature, 41, 478-539

Poon, W.P.H. \& Shen, J. (2020). The roles of rating outlooks: the predictor of creditworthiness and the monitor of recovery efforts. Rev.Quant.Finan.Acc., (2020). https://doi.org/10.1007/s11156-019-00868-7

Stigler, G.J., \& Kindahl, J.K. (1970). The Dispersion of Price Movements, in Ed. Stigler,G.J. \& Kindahl, J.K. The Behavior of Industrial Prices, NBER, 88 - 94

Schuermann, T. \& Jafry, Y. (2003). Measurement and Estimation of Credit Migration Matrices, The Wharton Financial Institutions Center, 1-44

Schwert, G.W. (1988). Why Does Stock Market Volatility Change Over Time? NBER WP 2798.

Shephard, N.G. (1991). From Characteristic Function to Distribution Function: A Simple Framework for the Theory. Econometric Theory, 7 (4), 519-529

Shiryaev, A.N. (1999). Essentials Of Stochastic Finance: Facts, Models, Theory. World Sc. Pub., Singapore. 1-852

S\&P, (2014). Guide To Credit Rating Essentials. What are credit ratings and how do they work? McGraw Hill Financial

S\&P\&, (2016). S\&P Global Ratings Definitions. S\&P Global Ratings

S\&P, (2018). 2018 Annual Global Corporate Default And Rating Transition Study

Tauchen, G.E. \& Pitts, M. (1983). The Price Variability-Volume Relationship On Speculative Markets, Econometrica, 51, (2), 485-505 


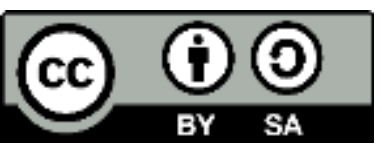

(C) 2021 by the authors. Licensee ACRN Publishing, Austria, Editor in Chief Prof. Dr. Othmar M. Lehner. This article is an open access article distributed under the terms and conditions of the Creative Commons Attribution (CC BY SA) license (https://creativecommons.org/licenses/by-sa/4.0/)

\section{Appendix A. Trades and their flows}

We define the collective second-degree trade $\boldsymbol{B}(2 ; t, z)$ between points $\boldsymbol{x}$ and $\boldsymbol{y}$ as a sum of all second-degree trades $\boldsymbol{b}_{i j}(2 ; t, \boldsymbol{z})$ of the agents $i$ with coordinates $\boldsymbol{x}$ in a unit volume $d V(\boldsymbol{x})$ and the agents $j$ with coordinates $\boldsymbol{y}$ in a unit volume $d V(y)(3.10)$ and then average this sum during the interval $\Delta$

$$
\begin{gathered}
\boldsymbol{B}(2 ; t, \mathbf{z})=\sum_{i \in d V(\boldsymbol{x}) ; j \in d V(\boldsymbol{y}) ; \Delta} \boldsymbol{b}_{i, j}(2 ; t, \mathbf{z}) \\
\sum_{i \in d V(\boldsymbol{x}) ; j \in d V(\boldsymbol{y}) ; \Delta} \boldsymbol{b}_{i, j}(2 ; t, \mathbf{z})=\frac{1}{\Delta} \int_{t-\Delta / 2}^{t+\Delta / 2} d \tau \sum_{i \in d V(\boldsymbol{x}) ; j \in d V(\boldsymbol{y})} \boldsymbol{b}_{i, j}(2 ; \tau, \mathbf{z}) \\
\boldsymbol{B}(2 ; t, \mathbf{z})=(U(2 ; t, \mathbf{z}) ; C(2 ; t, \mathbf{z})) ; \mathbf{z}=(\boldsymbol{x}, \boldsymbol{y}) \\
U(2 ; t, \mathbf{z})=\sum_{i \in d V(\boldsymbol{x}) ; j \in d V(\boldsymbol{y}) ; \Delta} U_{i j}^{2}(t, \mathbf{z}) \\
C(2 ; t, \boldsymbol{z})=\sum_{i \in d V(\boldsymbol{x}) ; j \in d V(\boldsymbol{y}) ; \Delta} C_{i j}^{2}(t, \mathbf{z})
\end{gathered}
$$

$U(2 ; t, z)$ and $C(2 ; t, z)($ A.3-A.5) denote the collective squares of the volume and the value of the trades between the points $\boldsymbol{x}$ and $\boldsymbol{y}$ averaged during the interval $\Delta$.

To describe the flows of the second-degree transactions $\boldsymbol{B}(2 ; t, z)$ let us assume that in the economic domain (3.2) the agent $i$ at the moment $t$ has the risk coordinates $\boldsymbol{x}=\left(x_{1}, \ldots x_{m}\right)$ and the velocities $\boldsymbol{v}_{\boldsymbol{x}}=\left(x_{y 1}, \ldots v_{x m}\right)$. Let us take that the agent $i$ at the point $\boldsymbol{x}$ executes the transaction $\boldsymbol{b}_{i j}(2 ; t, z)$ with the agent $j$ at the point $\boldsymbol{y}=\left(y_{1}, \ldots y_{m}\right)$ with the velocities $\boldsymbol{v}_{\boldsymbol{y}}=\left(v_{y 1}, \ldots v_{y m}\right)$. Similar to (Olkhov, 2018; 2019a - 2019c) let us define the flows $\boldsymbol{p}_{i j}(2 ; t, z)$ of the transactions $\boldsymbol{b}_{i j}(2 ; t, z)$ (3.7) between the agents $i$ and $j$ as:

$$
\begin{gathered}
\boldsymbol{p}_{i j}(2 ; t, \mathbf{z})=\left(\boldsymbol{p}_{U i j}(2 ; t, \mathbf{z}), \boldsymbol{p}_{C i j}(2 ; t, \mathbf{z})\right) \\
\boldsymbol{p}_{U i j}(2 ; t, \mathbf{z})=\left(\boldsymbol{p}_{U x i j}(2 ; t, \mathbf{z}) ; \boldsymbol{p}_{U y i j}(2 ; t, \mathbf{z})\right) ; \boldsymbol{p}_{C i j}(2 ; t, \mathbf{z})=\left(\boldsymbol{p}_{C x i j}(2 ; t, \mathbf{z}) ; \boldsymbol{p}_{C y i j}(2 ; t, \mathbf{z})\right) \\
\boldsymbol{p}_{U x i j}(2 ; t, \mathbf{z})=U_{i j}^{2}(t, \mathbf{z}) \boldsymbol{v}_{x i}(t, \boldsymbol{x}) ; \boldsymbol{p}_{U y i j}(t, \mathbf{z})=U_{i j}^{2}(t, \mathbf{z}) \boldsymbol{v}_{y j}(t, \boldsymbol{y}) \\
\boldsymbol{p}_{C x i j}(t, \mathbf{z})=C_{i j}^{2}(t, \mathbf{z}) \boldsymbol{v}_{x i}(t, \boldsymbol{x}) ; \boldsymbol{p}_{C i j y}(t, \mathbf{z})=C_{i j}^{2}(t, \mathbf{z}) \boldsymbol{v}_{y j}(t, \boldsymbol{y})
\end{gathered}
$$

The flows $\boldsymbol{p}_{i j}(2 ; t, z)$ (A.6) denote the flows $\boldsymbol{p}_{U i j}(2 ; t, z)\left(\right.$ A.8) that carry the square of the volume $U_{i j}{ }^{2}$ of the trade $\boldsymbol{b}_{i j}(2 ; t, \boldsymbol{z})$ (3.7). The flows $\boldsymbol{p}_{C i j}(t, z)$ (A.9) carry the square of the value $C_{i j}{ }^{2}$ of the trade $\boldsymbol{b}_{i j}(2 ; t, z)(3.7)$. We determine the cumulative flows $\boldsymbol{P}(2 ; t, \boldsymbol{z})$ of the macro second-degree transaction $\boldsymbol{B}(2 ; t, \boldsymbol{z}), \boldsymbol{z}=(\boldsymbol{x}, \boldsymbol{y})$ between the points $\boldsymbol{x}$ and $\boldsymbol{y}$ similar to (A.1-A.5) as the sums of flows $\boldsymbol{p}_{i j}(2 ; t, z)$ (A.6) of all second-degree transactions $\boldsymbol{b}_{i j}(2 ; t, z)(3.7)$ between the agents in a small volumes $d V(\boldsymbol{x})$ and $d V(\boldsymbol{y})(3.10)$ in the economic domain $(3.4,3.5)$ and averaging during an interval $\Delta$. Due to (A.1-A.5) we introduce the flows $\boldsymbol{P}(2 ; t, \boldsymbol{z})$ and the velocities $\boldsymbol{v}(2 ; t, \boldsymbol{z})$ as:

$$
\begin{aligned}
& \boldsymbol{P}(2 ; t, \mathbf{z})=\left(\boldsymbol{P}_{U}(2 ; t, \boldsymbol{z}), \boldsymbol{P}_{C}(2 ; t, \boldsymbol{z})\right) ; \boldsymbol{z}=(\boldsymbol{x}, \boldsymbol{y}) \\
& \boldsymbol{P}_{U}(2 ; t, \boldsymbol{z})=\sum_{i \in d V(\boldsymbol{x}) ; j \in d V(\boldsymbol{y}) \Delta} \boldsymbol{p}_{U i j}(2 ; t, \boldsymbol{z}) \\
& \boldsymbol{P}_{C}(t, \mathbf{z})=\sum_{i \in d V(x) ; j \in d V(\boldsymbol{y}) \Delta} \boldsymbol{p}_{C i j}(2 ; t, \mathbf{z}) \\
& \boldsymbol{P}_{U}(2 ; t, \mathbf{z})=\left(\boldsymbol{P}_{U x}(t, \mathbf{z}) ; \boldsymbol{P}_{U y}(t, \mathbf{z})\right) ; \boldsymbol{P}_{C}(t, \mathbf{z})=\left(\boldsymbol{P}_{C x}(t, \mathbf{z}) ; \boldsymbol{P}_{C y}(t, \mathbf{z})\right) \\
& \boldsymbol{P}_{U x}(2 ; t, \boldsymbol{z})=\sum_{i \in d V(\boldsymbol{x}) ; j \in d V(\boldsymbol{y}) \Delta} U_{i j}^{2}(t, \boldsymbol{z}) \boldsymbol{v}_{i}(t, \boldsymbol{x})=U(2 ; t, \boldsymbol{z}) \boldsymbol{v}_{U x}(t, \boldsymbol{z}) \\
& \boldsymbol{P}_{U y}(2 ; t, \mathbf{z})=\sum_{i \in d V(\boldsymbol{x}) ; j \in d V(\boldsymbol{y}) \Delta} U_{i j}^{2}(t, \mathbf{z}) \boldsymbol{v}_{j}(t, \boldsymbol{y})=U(2 ; t, \mathbf{z}) \boldsymbol{v}_{U y}(t, \mathbf{z}) \\
& \boldsymbol{P}_{C x}(2 ; t, \mathbf{z})=\sum_{i \in d V(\boldsymbol{x}) ; j \in d V(\boldsymbol{y}) \Delta} C_{i j}^{2}(t, \mathbf{z}) \boldsymbol{v}_{i}(t, \boldsymbol{x})=C(2 ; t, \mathbf{z}) \boldsymbol{v}_{C X}(t, \mathbf{z}) \\
& \boldsymbol{P}_{C y}(2 ; t, \boldsymbol{z})=\sum_{i \in d V(\boldsymbol{x}) ; j \in d V(\boldsymbol{y}) \Delta} C_{i j}^{2}(t, \boldsymbol{z}) \boldsymbol{v}_{j}(t, \boldsymbol{y})=C(2 ; t, \boldsymbol{z}) \boldsymbol{v}_{C y}(t, \boldsymbol{z}) \\
& \boldsymbol{v}(2 ; t, \mathbf{z})=\left(\boldsymbol{v}_{U}(2 ; t, \boldsymbol{z}) ; \boldsymbol{v}_{C}(2 ; t, \mathbf{z})\right) \\
& \boldsymbol{v}_{U}(2 ; t, \mathbf{z})=\left(\boldsymbol{v}_{U x}(2 ; t, \mathbf{z}) ; \boldsymbol{v}_{U y}(2 ; t, \mathbf{z})\right) ; \boldsymbol{v}_{C}(2 ; t, \mathbf{z})=\left(\boldsymbol{v}_{C x}(2 ; t, \mathbf{z}) ; \boldsymbol{v}_{C y}(2 ; t, \mathbf{z})\right)
\end{aligned}
$$

The integral of the second-degree trade $\boldsymbol{B}(2 ; t, z)$ (A.1-A.5) by $d \boldsymbol{y}$ over the economic domain (3.2) determines all selltrades $\boldsymbol{B}_{s}(2 ; t, \boldsymbol{x})$ from point $\boldsymbol{x}$ :

$$
\begin{aligned}
& \boldsymbol{B}_{\boldsymbol{s}}(2 ; t, \boldsymbol{x})=\int d \boldsymbol{y} \boldsymbol{B}(2 ; t, \boldsymbol{x}, \boldsymbol{y})=\left(U_{s}(2 ; t, \boldsymbol{x}) ; C_{s}(2 ; t, \boldsymbol{x})\right) \\
& U_{s}(2 ; t, \boldsymbol{x})=\int d \boldsymbol{y} U(2 ; t, \boldsymbol{x}, \boldsymbol{y}) ; C_{s}(2 ; t, \boldsymbol{x})=\int d \boldsymbol{y} C(2 ; t, \boldsymbol{x}, \boldsymbol{y})
\end{aligned}
$$

The integral $\boldsymbol{B}(2 ; t, z)$ by $d \boldsymbol{x}$ over the economic domain (3.2) determines all buy-trades $\boldsymbol{B}_{\boldsymbol{b}}(2 ; t, \boldsymbol{y})$ at $\boldsymbol{y}$

$$
\begin{gathered}
\boldsymbol{B}_{\boldsymbol{b}}(2 ; t, \boldsymbol{y})=\int d \boldsymbol{x} \boldsymbol{B}(2 ; t, \boldsymbol{x}, \boldsymbol{y})=\left(U_{b}(2 ; t, \boldsymbol{y}) ; C_{b}(2 ; t, \boldsymbol{y})\right) \\
U_{b}(2 ; t, \boldsymbol{y})=\int d \boldsymbol{x} U(2 ; t, \boldsymbol{x}, \boldsymbol{y}) ; C_{b}(2 ; t, \boldsymbol{y})=\int d \boldsymbol{x} C(2 ; t, \boldsymbol{x}, \boldsymbol{y})
\end{gathered}
$$


The integral of the second-degree trade $B(2 ; t, z)$ by $d z=d x d y$ over the economic domain (3.2) defines all macro second-degree trades $\boldsymbol{B}(2 ; t)$ as a function of time $t$ only:

$$
\begin{aligned}
& \boldsymbol{B}(2 ; t)=\int d \boldsymbol{z} \boldsymbol{B}(2 ; t, \boldsymbol{z})=(U(2 ; t) ; C(2 ; t)) \\
& U(2 ; t)=\int d \mathbf{z} U(2 ; t, \mathbf{z}) ; C(2 ; t)=\int d \boldsymbol{z} C(2 ; t, \mathbf{z})
\end{aligned}
$$

The relations (A.19-A.24) introduce the flows and the velocities for the corresponding second-degree trades. Due to (A.14; A.15) obtain:

$$
\begin{gathered}
\boldsymbol{P}_{U x}(2 ; t, \boldsymbol{x})=\int d \boldsymbol{y} \boldsymbol{P}_{U x}(2 ; t, \boldsymbol{x}, \boldsymbol{y})=\sum_{i \in d V(x) ; j \in d V(\boldsymbol{y}) \Delta} \int d \boldsymbol{y} U_{i j}^{2}(t, \boldsymbol{x}, \boldsymbol{y}) \boldsymbol{v}_{i}(t, \boldsymbol{x}) \\
\boldsymbol{P}_{U x}(2 ; t, \boldsymbol{x})=\int d \boldsymbol{y} U(2 ; t, \boldsymbol{z}) \boldsymbol{v}_{U x}(2 ; t, \boldsymbol{z})=U_{S}(2 ; t, \boldsymbol{x}) \boldsymbol{v}_{U s x}(2 ; t, \boldsymbol{x}) \\
\boldsymbol{P}_{U y}(2 ; t, \boldsymbol{x})=\int d \boldsymbol{y} \boldsymbol{P}_{U y}(2 ; t, \boldsymbol{x}, \boldsymbol{y})=\sum_{i \in d V(\boldsymbol{x}) ; j \in d V(\boldsymbol{y}) \Delta \int d \boldsymbol{y} U_{i j}^{2}(t, \boldsymbol{x}, \boldsymbol{y}) \boldsymbol{v}_{j}(t, \boldsymbol{y})} \\
\boldsymbol{P}_{U y}(2 ; t, \boldsymbol{x})=\int d \boldsymbol{y} U(2 ; t, \boldsymbol{z}) \boldsymbol{v}_{U y}(2 ; t, \boldsymbol{z})=U_{S}(2 ; t, \boldsymbol{x}) \boldsymbol{v}_{U b y}(2 ; t, \boldsymbol{x})
\end{gathered}
$$

The relations (A.25, A.26) introduce the aggregate flow of the sales $\boldsymbol{P}_{U x}(2 ; t, \boldsymbol{x})$ of the second-degree volume $U_{s}(2 ; t, \boldsymbol{x})$ and the collective velocity $\boldsymbol{v}_{U s x}(2 ; t, \boldsymbol{x})$ of the sellers at point $\boldsymbol{x}$ along axis $\mathrm{X}$ to all buyers in the economy. The relations (A.27, A.28) introduce the aggregate flow of the sales $\boldsymbol{P}_{U y}(2 ; t, \boldsymbol{x})$ of the second-degree volume $U_{s}(2 ; t, \boldsymbol{x})$ and the collective velocity $\boldsymbol{v}_{U s y}(2 ; t, \boldsymbol{x})$ of all buyers in the economy along the axis Y from the collective sellers at point $\boldsymbol{x}$. Corresponding relations for the second-degree value flows $\boldsymbol{P}_{C s x}(2 ; t, \boldsymbol{x})$ and $\boldsymbol{P}_{C s y}(2 ; t, \boldsymbol{x})$ take form:

$$
\begin{aligned}
& \boldsymbol{P}_{C s x}(2 ; t, \boldsymbol{x})=\int d \boldsymbol{y} \boldsymbol{P}_{C x}(2 ; t, \boldsymbol{x}, \boldsymbol{y})=\sum_{i \in d V(\boldsymbol{x}) ; j \in d V(\boldsymbol{y}) \Delta} \int d \boldsymbol{y} C_{i j}^{2}(t, \boldsymbol{x}, \boldsymbol{y}) \boldsymbol{v}_{i}(t, \boldsymbol{x}) \\
& \boldsymbol{P}_{C s x}(2 ; t, \boldsymbol{x})=C_{s}(2 ; t, \boldsymbol{x}) \boldsymbol{v}_{C s x}(2 ; t, \boldsymbol{x}) \\
& \boldsymbol{P}_{C s y}(2 ; t, \boldsymbol{x})=\int d \boldsymbol{y} \boldsymbol{P}_{C y}(2 ; t, \boldsymbol{x}, \boldsymbol{y})=\sum_{i \in d V(x) ; j \in d V(\boldsymbol{y}) \Delta} \int d \boldsymbol{y} C_{i j}^{2}(t, \boldsymbol{x}, \boldsymbol{y}) \boldsymbol{v}_{j}(t, \boldsymbol{y}) \\
& \boldsymbol{P}_{C s y}(2 ; t, \boldsymbol{x})=C_{s}(2 ; t, \boldsymbol{x}) \boldsymbol{v}_{C s y}(2 ; t, \boldsymbol{x})
\end{aligned}
$$

The similar relations define the buyers' flows. To derive the buyers' second-degree volume and the value flows $\boldsymbol{P}_{U b x}(2 ; t, \boldsymbol{y})$ and $\boldsymbol{P}_{U b y}(2 ; t, \boldsymbol{y}), \boldsymbol{P}_{C b x}(2 ; t, \boldsymbol{y})$ and $\boldsymbol{P}_{C b y}(2 ; t, \boldsymbol{y})$ one should take the integral for (A.15-A.18) by $d \boldsymbol{x}$ over the economic domain $(3.4 ; 3.5)$. For brevity we omit it here. The integrals by $d \boldsymbol{x} d \boldsymbol{y}$ for (A.14-A.18) over the entire economy - over the economic domain $(3.4 ; 3.5)$ introduce macroeconomic flows of the second-degree trades as functions of time $t$ only:

$$
\begin{aligned}
& \boldsymbol{P}_{U x}(2 ; t)=\int d \boldsymbol{z} \boldsymbol{P}_{U x}(2 ; t, \boldsymbol{z})=\sum_{i \in d V(x) ; j \in d V(\boldsymbol{y}) \Delta} \int d \boldsymbol{x} d \boldsymbol{y} U_{i j}^{2}(t, \boldsymbol{x}, \boldsymbol{y}) \boldsymbol{v}_{i}(t, \boldsymbol{x}) \\
& \boldsymbol{P}_{U x}(2 ; t)=U(2 ; t) \boldsymbol{v}_{U x}(2 ; t) \\
& \boldsymbol{P}_{U y}(2 ; t)=\int d \boldsymbol{z} \boldsymbol{P}_{U y}(2 ; t, \boldsymbol{z})=\sum_{i \in d V(\boldsymbol{x}) ; j \in d V(\boldsymbol{y}) \Delta} \int d \boldsymbol{x} d \boldsymbol{y} U_{i j}^{2}(t, \boldsymbol{x}, \boldsymbol{y}) \boldsymbol{v}_{j}(t, \boldsymbol{y}) \\
& \boldsymbol{P}_{U y}(2 ; t)=U(2 ; t) \boldsymbol{v}_{U y}(2 ; t) \\
& \boldsymbol{P}_{C x}(2 ; t)=\int d \boldsymbol{z} \boldsymbol{P}_{C x}(2 ; t, \boldsymbol{z})=\sum_{i \in d V(\boldsymbol{x}) ; j \in d V(\boldsymbol{y}) \Delta} \int d \boldsymbol{x} d \boldsymbol{y} C_{i j}^{2}(t, \boldsymbol{x}, \boldsymbol{y}) \boldsymbol{v}_{i}(t, \boldsymbol{x}) \\
& \boldsymbol{P}_{C x}(2 ; t)=C(2 ; t) \boldsymbol{v}_{C x}(2 ; t) \\
& \boldsymbol{P}_{C y}(2 ; t)=\int d \boldsymbol{z} \boldsymbol{P}_{C y}(2 ; t, \boldsymbol{z})=\sum_{i \in d V(\boldsymbol{x}) ; j \in d V(\boldsymbol{y}) \Delta} \int d \boldsymbol{x} d \boldsymbol{y} C_{i j}^{2}(t, \boldsymbol{x}, \boldsymbol{y}) \boldsymbol{v}_{j}(t, \boldsymbol{y}) \\
& \boldsymbol{P}_{C y}(2 ; t)=C(2 ; t) \boldsymbol{v}_{C y}(2 ; t)
\end{aligned}
$$

The relations (A.33-A.40) introduce a new economic concept - the collective flows of all agents of the economy involved into the second-degree trades in the economic domain $(3.4,3.5)$. The collective flows of the second-degree volume $\left(\boldsymbol{P}_{U x}(2 ; t) ; \boldsymbol{P}_{U y}(2 ; t)\right)$, and the flows of the second-degree value $\left(\boldsymbol{P}_{C x}(2 ; t) ; \boldsymbol{P}_{C y}(2 ; t)\right)$ define the corresponding collective velocities of the volume $\left(\boldsymbol{v}_{U x}, \boldsymbol{v}_{U y}\right)$ and the value $\left(\boldsymbol{v}_{C x}, \boldsymbol{v}_{C y}\right)$. Velocity $\boldsymbol{v}_{U x}$ defines the collective motion of all sellers in the economy weighted by the volumes of the trades during $\Delta$. Velocity $\boldsymbol{v}_{U y}$ defines collective motion of all buyers in the economy weighted by the volumes of the trades during $\Delta$. Velocities $\left(\boldsymbol{v}_{C x}, \boldsymbol{v}_{C y}\right)$ define collective motion of all sellers and all buyers weighted by the value of all trades during $\Delta$. These velocities describe the collective motion of the entire economy in the economic domain $(3.4,3.5)$ reduced by its borders at 0 and 1 by all axes. Hence, these collective velocities cannot have the constant direction and must oscillate. The collective velocities of the volume $\left(\boldsymbol{v}_{U x}, \boldsymbol{v}_{U y}\right)$ and the value $\left(\boldsymbol{v}_{C x}, \boldsymbol{v}_{C y}\right)$ occasionally change their direction and describe the collective motion of the sellers and the buyers from the secure area of the economic domain to the risky one and back. We suggest that such fluctuations of the second-degree trades accompanied with rise and fall of the market activity, production functions, demand and supply, price variations, etc., establish the ground for the economic fluctuations of the business cycles. We describe the motion of the collective trades in the economic domain $(3.4,3.5)$ as hidden properties of the business cycles (Olkhov, 2017b, 2019a). 


\section{Appendix B. Collective expectations}

To define the collective expectations we introduce a new economic issue: the collective expected trades. To do that we use (4.1) to define the collective second-degree trades $U(2, \boldsymbol{k} ; t, \boldsymbol{z}), C(2, \boldsymbol{k} ; t, \boldsymbol{z})$ approved by the sellers' expectations of type $\boldsymbol{k}=(k, l)$ as:

$$
\begin{aligned}
& U(2, \boldsymbol{k} ; t, \mathbf{z})=\sum_{i \in d V(\boldsymbol{x}) ; j \in d V(\boldsymbol{y}) \Delta} U_{i j}^{2}(\boldsymbol{k} ; t, \mathbf{z}) \\
& C(2, \boldsymbol{k} ; t, \mathbf{z})=\sum_{i \in d V(\boldsymbol{x}) ; j \in d V(\boldsymbol{y}) \Delta C_{i j}^{2}(\boldsymbol{k} ; t, \mathbf{z})}
\end{aligned}
$$

Definitions of the collective flows $\boldsymbol{P}_{U}(2, \boldsymbol{k} ; t, \boldsymbol{z})$ and $P_{C}(2, \boldsymbol{k} ; t, \boldsymbol{z})$ of the trades with the volume $U(2, \boldsymbol{k} ; t, \boldsymbol{z})$ and the value $C(2, \boldsymbol{k} ; t, \boldsymbol{z})($ B.1,B.2) are similar to (A.25-A.28) and we omit them here for brevity. The trades $U(2, \boldsymbol{k} ; t, \boldsymbol{z}), C(2, \boldsymbol{k} ; t, \boldsymbol{z})$ (B.1, B.2) follow the obvious relations (A.1-A.5):

$$
U(2 ; t, \mathbf{z})=\sum_{k, l=1}^{K} U(2, \boldsymbol{k} ; t, \mathbf{z}) ; C(2 ; t, \mathbf{z})=\sum_{k, l=1}^{K} C(2, \boldsymbol{k} ; t, \mathbf{z})
$$

The collective second-degree trades $U(2 ; t, z ; l), C(2 ; t, z ; l)$ approved by the buyers' expectations take form:

$$
\begin{aligned}
& U(2 ; t, \boldsymbol{z} ; \boldsymbol{l})=\sum_{i \in d V(\boldsymbol{x}) ; j \in d V(\boldsymbol{y}) \Delta} U_{i j}^{2}(t, \boldsymbol{z} ; \boldsymbol{l}) \\
& C(2 ; t, \boldsymbol{z} ; \boldsymbol{l})=\sum_{i \in d V(\boldsymbol{x}) ; j \in d V(\boldsymbol{y}) \Delta} C_{i j}^{2}(t, \boldsymbol{z} ; \boldsymbol{l})
\end{aligned}
$$

The buyers' trades obey the relations alike to (B.3). Similar to (A.1-A.5) we use (4.5-4.7) and introduce the sellers' collective expected trades $\boldsymbol{E} t(\boldsymbol{k} ; t, \boldsymbol{z})$ as:

$$
\begin{gathered}
\boldsymbol{E} \boldsymbol{t}(k ; t, \mathbf{z})=\left(E t_{U}(\boldsymbol{k} ; t, \mathbf{z}) ; E t_{C}(\boldsymbol{k} ; t, \boldsymbol{z})\right) \\
E t_{U}(\boldsymbol{k} ; t, \mathbf{z})=\sum_{i \in d V(\boldsymbol{x}) ; j \in d V(\boldsymbol{y}) ; \Delta} \operatorname{ex}_{U i}(\boldsymbol{k} ; t, \boldsymbol{x}) U_{i j}^{2}(\boldsymbol{k} ; t, \mathbf{z}) \\
E t_{C}(\boldsymbol{k} ; t, \mathbf{z})=\sum_{i \in d V(\boldsymbol{x}) ; j \in d V(\boldsymbol{y}) ; \Delta} \operatorname{ex}_{C i}(\boldsymbol{k} ; t, \boldsymbol{x}) C_{i j}^{2}(\boldsymbol{k} ; t, \mathbf{z})
\end{gathered}
$$

The buyers' collective second-degree expected trades $\boldsymbol{E} \boldsymbol{t}(t, z ; \boldsymbol{l})$,

$$
\begin{gathered}
\boldsymbol{E t}(t, \boldsymbol{z} ; \boldsymbol{l})=\left(E t_{U}(t, \boldsymbol{z} ; \boldsymbol{l}) ; E t_{C}(t, \boldsymbol{z} ; \boldsymbol{l})\right) \\
E t_{U}(t, \boldsymbol{z} ; \boldsymbol{l})=\sum_{i \in d V(\boldsymbol{x}) ; j \in d V(\boldsymbol{y}) ; \Delta} \operatorname{ex}_{U j}(t, \boldsymbol{y} ; \boldsymbol{l}) U_{i j}^{2}(t, \boldsymbol{z} ; \boldsymbol{l}) \\
E t_{C}(t, \boldsymbol{z} ; \boldsymbol{l})=\sum_{i \in d V(\boldsymbol{x}) ; j \in d V(\boldsymbol{y}) ; \Delta} \operatorname{ex}_{C j}(t, \boldsymbol{y} ; \boldsymbol{l}) C_{i j}^{2}(t, \boldsymbol{z} ; \boldsymbol{l})
\end{gathered}
$$

The relations (B.6-B.8) and (B.1; B.2) introduce the collective sellers' expectations $\boldsymbol{E x}(\boldsymbol{k} ; t, \boldsymbol{z})$, as:

$$
\begin{aligned}
& E x(\boldsymbol{k} ; t, \mathbf{z})=\left(E x_{U}(\boldsymbol{k} ; t, \mathbf{z}), E x_{C}(\boldsymbol{k} ; t, \mathbf{z})\right) \\
& E x_{U}(\boldsymbol{k} ; t, \mathbf{z}) U(2, \boldsymbol{k} ; t, \mathbf{z})=E t_{U}(\boldsymbol{k} ; t, \mathbf{z}) \\
& E x_{C}(\boldsymbol{k} ; t, \mathbf{z}) C(2, \boldsymbol{k} ; t, \mathbf{z})=E t_{C}(\boldsymbol{k} ; t, \mathbf{z})
\end{aligned}
$$

The collective buyers' expectations take similar form:

$$
\begin{aligned}
& E x(t, \mathbf{z} ; \boldsymbol{l})=\left(E x_{U}(t, \mathbf{z} ; \boldsymbol{l}), E x_{C}(t, \mathbf{z} ; \boldsymbol{l})\right) \\
& E x_{U}(t, \mathbf{z} ; \boldsymbol{l}) U(2 ; t, \mathbf{z} ; \boldsymbol{l})=E t_{U}(t, \mathbf{z} ; \boldsymbol{l}) \\
& E x_{C}(t, \mathbf{z} ; \boldsymbol{l}) C(2 ; t, \mathbf{z} ; \boldsymbol{l})=E t_{C}(t, \mathbf{z} ; \boldsymbol{l})
\end{aligned}
$$

Let us take the integrals over the economic domain $(3.4,3.5)$ of $U(2, \boldsymbol{k} ; t, z), C(2, \boldsymbol{k} ; t, z)($ B.1, B.2):

$$
U(2, \boldsymbol{k} ; t)=\int d \boldsymbol{z} U(2, \boldsymbol{k} ; t, \mathbf{z}) ; C(2, \boldsymbol{k} ; t)=\int d \boldsymbol{z} C(2, \boldsymbol{k} ; t, \mathbf{z})
$$

The relations (B.18) define the collective sellers' second-degree trades $U(2, \boldsymbol{k} ; t), C(2, \boldsymbol{k} ; t)$ performed under the expectations of the type $\boldsymbol{k}=(k, l)$. Then the integral by the economic domain $(3.4,3.5)$ of the expected trades (B.13, B.14) define the collective sellers' expected trades $E t_{U}(\boldsymbol{k} ; t), E t_{C}(\boldsymbol{k} ; t)$ of the entire economy

$$
E t_{U}(\boldsymbol{k} ; t)=\int d \boldsymbol{z} E t_{U}(\boldsymbol{k} ; t, \mathbf{z}) ; E t_{C}(\boldsymbol{k} ; t)=\int d \mathbf{z} E t_{C}(\boldsymbol{k} ; t, \mathbf{z})
$$

(B.18, B.19) define collective sellers' expectations $E x_{U}(\boldsymbol{k} ; t), E x_{C}(\boldsymbol{k} ; t)$ of the entire economy

$$
\begin{gathered}
E x_{U}(\boldsymbol{k} ; t) U(2, \boldsymbol{k} ; t)=E t_{U}(\boldsymbol{k} ; t) \\
E x_{C}(\boldsymbol{k} ; t) C(2, \boldsymbol{k} ; t)=E t_{C}(\boldsymbol{k} ; t)
\end{gathered}
$$

Similar relations define the collective buyers' expected trades $E t_{U}(t ; \boldsymbol{l}), E t_{C}(t ; \boldsymbol{l})$ and the expectations $E x_{U}(t ; \boldsymbol{l}), E x_{C}(t ; \boldsymbol{l})$ of the entire economy:

$$
\begin{aligned}
& E x_{U}(t ; \boldsymbol{l}) U(2 ; t ; \boldsymbol{l})=E t_{U}(t ; \boldsymbol{l}) \\
& E x_{C}(t ; \boldsymbol{l}) C(2 ; t ; \boldsymbol{l})=E t_{C}(t ; \boldsymbol{l})
\end{aligned}
$$

The relations (B.20) introduce the collective sellers' expected trades $E t_{U s}(t), E t_{C s}(t)$ :

$$
E t_{U s}(t)=\sum_{k, l=1}^{K} E t_{U}(\boldsymbol{k} ; t) ; E t_{C S}(t)=\sum_{k, l=1}^{K} E t_{C}(\boldsymbol{k} ; t)
$$

The relations (B.24) define the collective sellers' expectations $E x_{U s}(t), E x_{C s}(t)$ :

$$
E x_{U S}(t) U(2 ; t)=E t_{U S}(t) ; E x_{C S}(t) C(2 ; t)=E t_{C S}(t)
$$

Similar relations define the collective buyers' expectations of the entire economy. We omit them for brevity. It is clear that the collective sellers' expectations those approve the trades with the squares of the volume $U^{2}$ and the squares of the value $C^{2}$ may be different. The collective sellers' expectations may be different from the buyers' expectations. Such diversity uncovers the hidden complexity of the mutual interactions between the transactions and 
the expectations. The long set of the above definitions outlines multiplicity of the different states of the collective trades and the expectations. One easy defines the collective flows of sellers' and buyers' expected trades similar to (Olkhov, 2019b). For brevity we present here only definition of the sellers' volume flow of the expected trades $\boldsymbol{P} \boldsymbol{e}_{U x}$, $\boldsymbol{P e}_{U y}$ in (B.26-B2.9):

$$
\begin{aligned}
& \boldsymbol{p e}_{U x i j}(\boldsymbol{k} ; t, \mathbf{z})=e t_{U i j}(\boldsymbol{k} ; t, \mathbf{z}) \boldsymbol{v}_{i}(t, \boldsymbol{x})=e x_{i}(\boldsymbol{k} ; t, \boldsymbol{x}) U_{i j}^{2}(\boldsymbol{k} ; t, \mathbf{z}) \boldsymbol{v}_{i}(t, \boldsymbol{x}) \\
& \boldsymbol{p e}_{U y i j}(\boldsymbol{k} ; t, \mathbf{z})=e t_{U i j}(\boldsymbol{k} ; t, \mathbf{z}) \boldsymbol{v}_{j}(t, \boldsymbol{y})=\operatorname{ex}_{i}(\boldsymbol{k} ; t, \boldsymbol{x}) U_{i j}^{2}(\boldsymbol{k} ; t, \mathbf{z}) \boldsymbol{v}_{j}(t, \boldsymbol{y}) \\
& \boldsymbol{P e}_{U x}(\boldsymbol{k} ; t, \mathbf{z})=\sum_{i \in d V(x) ; j \in d V(\boldsymbol{y}) \Delta} e t_{U i j}(\boldsymbol{k} ; t, \mathbf{z}) \boldsymbol{v}_{i}(t, \boldsymbol{x})=E t_{U}(\boldsymbol{k} ; t, \mathbf{z}) \boldsymbol{v} \boldsymbol{e}_{U x}(\boldsymbol{k} ; t, \mathbf{z}) \\
& \boldsymbol{P} \boldsymbol{e}_{U y}(\boldsymbol{k} ; t, \mathbf{z})=\sum_{i \in d V(\boldsymbol{x}) ; j \in d V(\boldsymbol{y}) \Delta} e t_{U i j}(\boldsymbol{k} ; t, \mathbf{z}) \boldsymbol{v}_{j}(t, \boldsymbol{y})=E t_{U}(\boldsymbol{k} ; t, \mathbf{z}) \boldsymbol{v} \boldsymbol{e}_{U y}(\boldsymbol{k} ; t, \mathbf{z})
\end{aligned}
$$

We do not introduce here the full set of the flows and refer (Olkhov, 2019b) for further detail.

\section{Appendix C. Equations on the second-degree trades}

We present here only equations for the second-degree trades $\boldsymbol{B}(2 ; t, z)$ (A.1-A.5) and refer for details (Olkhov, 2018$2019 b)$. Equations for the second-degree volume $U(2, \boldsymbol{k} ; t, \boldsymbol{z})$ and the value $C(2, \boldsymbol{k} ; \boldsymbol{t}, \boldsymbol{z})($ B.1; B.2) take form:

$$
\begin{aligned}
& \frac{\partial}{\partial t} U(2, \boldsymbol{k} ; t, \mathbf{z})+\nabla \cdot\left(U(2, \boldsymbol{k} ; t, \mathbf{z}) \boldsymbol{v}_{U}(2, \boldsymbol{k} ; t, \mathbf{z})\right)=F_{U}(\boldsymbol{k} ; t, \mathbf{z}) \\
& \frac{\partial}{\partial t} C(2, \boldsymbol{k} ; t, \mathbf{z})+\nabla \cdot\left(C(2, \boldsymbol{k} ; t, \mathbf{z}) \boldsymbol{v}_{C}(2, \boldsymbol{k} ; t, \mathbf{z})\right)=F_{C}(\boldsymbol{k} ; t, \mathbf{z})
\end{aligned}
$$

Equations on the flows $\boldsymbol{P}_{U x}(2 ; t, z)$ (A.15) of the second-degree volume $U(2 ; t, z)$ take form:

$$
\frac{\partial}{\partial t} \boldsymbol{P}_{U x}(\boldsymbol{k} ; t, \mathbf{z})+\nabla \cdot\left(\boldsymbol{P}_{U x}(\boldsymbol{k} ; t, \mathbf{z}) \boldsymbol{v}_{U x}(\boldsymbol{k} ; t, \mathbf{z})\right)=\boldsymbol{G}_{U x}(\boldsymbol{k} ; t, \mathbf{z})
$$

Equations on the flows (A.16-A.18) have similar form. The similar equations are valid for the expected trades $E x_{U}(\boldsymbol{k} ; t, z)\left(\right.$ B.6-B.8) and their flows $\boldsymbol{P} \boldsymbol{e}_{U x}(\boldsymbol{k} ; t, \mathbf{z})$ :

$$
\begin{aligned}
& \frac{\partial}{\partial t} E t_{U}(\boldsymbol{k} ; t, \mathbf{z})+\nabla \cdot\left(E t_{U}(\boldsymbol{k} ; t, \mathbf{z}) \boldsymbol{v} \boldsymbol{e}_{U x}(\boldsymbol{k} ; t, \boldsymbol{z})\right)=W_{U}(\boldsymbol{k} ; t, \mathbf{z}) \\
& \frac{\partial}{\partial t} \boldsymbol{P} \boldsymbol{e}_{U x}(\boldsymbol{k} ; t, \boldsymbol{z})+\nabla \cdot\left(\boldsymbol{P} \boldsymbol{e}_{U x}(\boldsymbol{k} ; t, \boldsymbol{z}) \boldsymbol{v} \boldsymbol{e}_{U x}(\boldsymbol{k} ; t, \boldsymbol{z})\right)=\boldsymbol{R}_{U x}(\boldsymbol{k} ; t, \boldsymbol{z})
\end{aligned}
$$

The integrals of the divergence by $d z$ over the domain $(3.4 ; 3.5)$ equal zero as no economic agents and no flows exist outside $(3.4 ; 3.5)$. Hence the integrals of (C.1-C.5) by $d z$ over domain $(3.4 ; 3.5)$ give:

$$
\begin{aligned}
& \int d \boldsymbol{z}\left[\frac{\partial}{\partial t} U(2, \boldsymbol{k} ; t, \mathbf{z})+\nabla \cdot\right.\left.\left(U(2, \boldsymbol{k} ; t, \mathbf{z}) \boldsymbol{v}_{U}(2, \boldsymbol{k} ; t, \mathbf{z})\right)\right]=\frac{d}{d t} U(2, \boldsymbol{k} ; t)=F_{U}(\boldsymbol{k} ; t)=\int d \boldsymbol{z} F_{U}(t, \mathbf{z}) \\
& \frac{d}{d t} C(2, \boldsymbol{k} ; t)=F_{C}(\boldsymbol{k} ; t) ; \quad \frac{d}{d t} \boldsymbol{P}_{U x}(\boldsymbol{k} ; t)=\boldsymbol{G}_{U x}(\boldsymbol{k} ; t) \\
& \frac{d}{d t} E t_{U}(\boldsymbol{k} ; t)=W_{U}(\boldsymbol{k} ; t) \quad ; \quad \frac{d}{d t} \boldsymbol{P} \boldsymbol{e}_{U x}(\boldsymbol{k} ; t)=\boldsymbol{R}_{U x}(\boldsymbol{k} ; t)
\end{aligned}
$$

(C.6-C.8) describe the equations on the collective second-degree trades, the expectations and their flows as functions of time $t$ and the type $\boldsymbol{k}$ of the expectations. Formal simplicity of (C.6-C.8) hides the complexity of the functions $F_{U}(t), F_{U}(t), \boldsymbol{G}_{U x}, \boldsymbol{W}_{U x}, \boldsymbol{R}_{U x}$, etc. These functions describe the impact of the expectations of the second-degree trades and their flows and the impact of the trades on the expected trades and their flows. These functions model the economic and the financial processes, the social expectations, the technology forecast, and the flows of these parameters those impact the second-degree trades $\boldsymbol{B}(2, t)(3.17)$ and the flows $\boldsymbol{P}(2, t)$ and back action of the trades on the expected trades and their flows. The integrals in the right side of (C.6-C.8) can describe the integrals by the flows and their products. These factors are completely new for the macroeconomic and financial theory and never before were taken into account. 


\section{Appendix D. Price volatility fluctuations}

Let us follow (Olkhov, 2019b) and neglect the impact of the trades and the expected trades flows. Let us study small perturbations of $U(2, \boldsymbol{k} ; t), C(2, \boldsymbol{k} ; t)$ and $E x_{U}(\boldsymbol{k} ; t)$ and $E x_{C}(\boldsymbol{k} ; t)$ as:

$$
\begin{aligned}
& U(2, \boldsymbol{k} ; t)=U_{\boldsymbol{k}}^{2}(1+u(2, \boldsymbol{k} ; t)) ; C(2, \boldsymbol{k} ; t)=C_{\boldsymbol{k}}^{2}(1+c(2, \boldsymbol{k} ; t)) \\
& E t_{U}(\boldsymbol{k} ; t)=E t_{U \boldsymbol{k}}\left(1+e t_{u}(\boldsymbol{k} ; t)\right) ; E t_{C}(\boldsymbol{k} ; t)=E t_{C \boldsymbol{k}}\left(1+e t_{c}(\boldsymbol{k} ; t)\right)
\end{aligned}
$$

The relations (D.1, D.2) describe small dimensionless disturbances $u, c$, et $_{u}$ et $_{c}$. The relations $(3.14,5.2$, D.1) present the second-degree volume $U(2 ; t)$, the value $C(2 ; t)$ and the price $p(2, t)(3.14)$ as:

$$
\begin{gathered}
U(2 ; t)=\sum_{k, l=1}^{K} U(2, \boldsymbol{k} ; t) ; C(2 ; t)=\sum_{k, l=1}^{K} C(2, \boldsymbol{k} ; t) ; C(2 ; t)=p(2 ; t) U(2 ; t) \\
p(2 ; t)=\frac{C(2 ; t)}{U(2 ; t)}=\frac{\sum_{k, l} C_{\boldsymbol{k}}^{2}(1+c(2, \boldsymbol{k} ; t))}{\sum_{k, l} U_{\boldsymbol{k}}^{2}(1+u(2, \boldsymbol{k} ; t))}
\end{gathered}
$$

In a linear approximation by the disturbances $u(2, \boldsymbol{k} ; t)$ and $c(2, \boldsymbol{k} ; t)$, the price $p(2 ; t)$ takes form:

$$
\begin{gathered}
p(2 ; t)=p_{20}[1+\pi(2 ; t)]=p_{20}\left[1+\sum_{k, l=1}^{K}\left(\mu_{2 \boldsymbol{k}} c(2, \boldsymbol{k} ; t)-\lambda_{2 k} u(2, \boldsymbol{k} ; t)\right)\right] \\
U_{20}=\sum_{k, l} U_{\boldsymbol{k}}^{2} ; C_{20}=\sum_{k, l} C_{\boldsymbol{k}}^{2} ; \quad C_{20}=p_{20} U_{20} \\
\lambda_{2 \boldsymbol{k}}=\frac{U_{\boldsymbol{k}}^{2}}{U_{20}} ; \quad \mu_{2 \boldsymbol{k}}=\frac{C_{\boldsymbol{k}}^{2}}{C_{20}} ; \sum \lambda_{2 \boldsymbol{k}}=\sum \mu_{2 \boldsymbol{k}}=1
\end{gathered}
$$

We use index 2 to underline that $p_{20}$ and other constants describe the properties of the price $p(2 ; t)$. Let us take into account (D.1) and present the disturbances of the price $p(2, \boldsymbol{k}, t)(5.2)$ as

Then in the linear approximation by disturbances obtain:

$$
C_{\boldsymbol{k}}^{2}[1+c(2, \boldsymbol{k} ; t)]=p_{2 k}[1+\pi(2, \boldsymbol{k} ; t)] U_{\boldsymbol{k}}^{2}[1+u(2, \boldsymbol{k} ; t)]
$$

$$
C_{\boldsymbol{k}}^{2}=p_{2 k} U_{\boldsymbol{k}}^{2} ; \pi(2 ; \boldsymbol{k} ; t)=c(2, \boldsymbol{k} ; t)-u(2, \boldsymbol{k} ; t)
$$

Now substitute (D.6) into (D.4) and obtain the dependence of the price disturbances $\pi(2 ; t)$ on the volume disturbances $u(2, \boldsymbol{k} ; t)$ :

$$
\begin{gathered}
\pi(2 ; t)=\sum_{k, l} \mu_{2 k} \pi(2, \boldsymbol{k} ; t)+\sum_{k, l}\left(\mu_{2 k}-\lambda_{2 k}\right) u(2, \boldsymbol{k} ; t) \\
p(2 ; t)=p_{20}[1+\pi(2 ; t)]=p_{20}\left[1+\sum_{k, l} \mu_{2 k} \pi(2, \boldsymbol{k} ; t)+\left(\mu_{2 k}-\lambda_{2 k}\right) u(2, \boldsymbol{k} ; t)\right]
\end{gathered}
$$

(D.7) describes the fluctuations $\pi(2 ; t)$ (D.4) of the mean square of price $p(2 ; t)$ as a function of partial square of the price disturbances $\pi(2, \boldsymbol{k} ; t)$ (D.6) and the square of the volume disturbances $u(2, \boldsymbol{k} ; t)$ (D.1). Now let us study the simple model that can describe the volume, the value and the price disturbances (D.1, D.4, D.8). Assume that $U_{k}{ }^{2}$, $C_{k}^{2}, E t_{U k}$ and $E t_{C k}$ in (D.1, D.2) are constant or their changes are slow to compare with the changes of small dimensionless disturbances $u(2, \boldsymbol{k} ; t), c(2, \boldsymbol{k} ; t), e t_{q}(\boldsymbol{k} ; t)$ and $e t_{c}(\boldsymbol{k} ; t)$. We assume that in the linear approximation by perturbations $F_{U}, F_{C}$ in (5.1) and $W_{U}, W_{C}$ in (5.5) take form:

$$
\begin{array}{cl}
F_{U}(\boldsymbol{k} ; t)=a_{u k} E t_{U k}\left(1+e t_{u}(\boldsymbol{k} ; t)\right) ; & F_{C}(\boldsymbol{k} ; t)=a_{c k} E t_{C \boldsymbol{k}}\left(1+e t_{c}(\boldsymbol{k} ; t)\right) \\
W_{U}(\boldsymbol{k} ; t)=b_{u k} U_{\boldsymbol{k}}^{2}(1+u(2, \boldsymbol{k} ; t)) ; & W_{C}(\boldsymbol{k} ; t)=b_{c k} C_{\boldsymbol{k}}^{2}(1+c(2, \boldsymbol{k} ; t))
\end{array}
$$

Taking into account (B.20, B.21) and (D.1, D.2) the equations $(5.1,5.6)$ in the linear approximation by disturbances take form:

For

$$
\begin{gathered}
U_{\boldsymbol{k}}^{2} \frac{d}{d t} u(2, \boldsymbol{k} ; t)=a_{u k} E t_{U \boldsymbol{k}} e t_{u}(\boldsymbol{k} ; t) ; \quad C_{\boldsymbol{k}}^{2} \frac{d}{d t} c(2, \boldsymbol{k} ; t)=a_{c k} E t_{C \boldsymbol{k}} e t_{c}(\boldsymbol{k} ; t) \\
E t_{U \boldsymbol{k}} \frac{d}{d t} e t_{U}(\boldsymbol{k} ; t)=b_{u k} U_{\boldsymbol{k}}^{2} u(2, \boldsymbol{k} ; t) ; \quad E t_{C \boldsymbol{k}} \frac{d}{d t} e t_{C}(\boldsymbol{k} ; t)=b_{c k} C_{\boldsymbol{k}}^{2} c(2, \boldsymbol{k} ; t)
\end{gathered}
$$

simple solutions for the disturbances $u(2, \boldsymbol{k} ; t), c(2, \boldsymbol{k} ; t), e t_{u}(\boldsymbol{k} ; t), e t_{c}(\boldsymbol{k} ; t)$ take the harmonic oscillations:

$$
\begin{aligned}
& u(2, \boldsymbol{k} ; t)=u_{1 k} \sin \omega_{u k} t+u_{2 k} \cos \omega_{u k} t \quad ; \quad u_{1 k}, u_{2 k} \ll 1 \\
& c(2, \boldsymbol{k} ; t)=c_{1 k} \sin \omega_{c k} t+c_{2 k} \cos \omega_{c k} t \quad ; \quad c_{1 k}, c_{2 k} \ll 1
\end{aligned}
$$

The solutions (D.8, D.9) are similar to the relations derived for the first-degree trades disturbances (Olkhov, 2019b). For the case

$$
\gamma_{u k}^{2}=-a_{u k} b_{u k}<0 ; \quad \gamma_{c k}^{2}=-a_{c k} b_{c k}<0
$$

Equations (D.11, D.12) describe the exponential growth or dissipations of the perturbations

$$
u(2, \boldsymbol{k} ; t) \sim \exp \gamma_{u k} t \quad ; \quad c(2, \boldsymbol{k} ; t) \sim \exp \gamma_{c k} t
$$

The perturbations $\pi(2, \boldsymbol{k} ; t)$ (D.6) may also follow the exponential growth or decline. For the different expectations $\boldsymbol{k}=(k, l)(4.1, \mathrm{~B} .7, \mathrm{~B} .8)$ the solutions for the disturbances $u(2, \boldsymbol{k} ; t), c(2, \boldsymbol{k} ; t)$ can take (D.14, D.15) or (D.17). The combinations of the perturbations determined by (D.14, D.15) or (D.17) define the perturbations of the price $p(2 ; t)$ 
(D.4). Thus simple equations $(5.1,5.5)$ on the second-degree volume and value may describe the unexpected exponential growth of the perturbations $\pi(2 ; t)$ (D.7) and hence, the exponential growth of the price $p(2 ; t)(\mathrm{D} .4 ;$ D.8) and the price volatility $\sigma_{p}^{2}(t)(2.17)$. Now let us assume that $u(2, \boldsymbol{k} ; t)$ and $c(2, \boldsymbol{k} ; t)$ disturbances depend on the disturbances of the expectations $e x_{u}(\boldsymbol{k} ; t), e x_{c}(\boldsymbol{k} ; t)$ (see B.20, B.21):

$$
E x_{U}(\boldsymbol{k} ; t)=E x_{U \boldsymbol{k}}\left(1+e x_{u}(\boldsymbol{k} ; t)\right) ; E x_{C}(\boldsymbol{k} ; t)=E x_{C \boldsymbol{k}}\left(1+e x_{C}(\boldsymbol{k} ; t)\right)
$$

Let us take into account (D.1) and in the linear approximation by the disturbances obtain:

$$
\begin{gathered}
E t_{U}(\boldsymbol{k} ; t)=E x_{U \boldsymbol{k}} U_{\boldsymbol{k}}^{2}\left(1+e x_{u}(\boldsymbol{k} ; t)+u(2, \boldsymbol{k} ; t)\right) \\
E t_{C}(\boldsymbol{k} ; t)=E x_{C \boldsymbol{k}} U_{\boldsymbol{k}}^{2}\left(1+e x_{C}(\boldsymbol{k} ; t)+c(2, \boldsymbol{k} ; t)\right)
\end{gathered}
$$

Now use (D.19, D.20) and replace (D.9) by (D.21, D.22):

$$
\begin{aligned}
& F_{U}(\boldsymbol{k} ; t)=a_{u k} E t_{U \boldsymbol{k}} U_{\boldsymbol{k}}^{2}\left(1+e x_{u}(\boldsymbol{k} ; t)+u(2, \boldsymbol{k} ; t)\right) \\
& F_{C}(\boldsymbol{k} ; t)=a_{c k} E t_{C \boldsymbol{k}} C_{\boldsymbol{k}}^{2}\left(1+e x_{c}(\boldsymbol{k} ; t)+c(2, \boldsymbol{k} ; t)\right)
\end{aligned}
$$

It is easy to show that for

$$
\begin{aligned}
& \omega_{u k}^{2}=-\frac{a_{u k} b_{u k}}{U_{k}}>0 ; \quad \omega_{c k}^{2}=-\frac{a_{c k} b_{c k}}{C_{k}}>0 \\
& \gamma_{u k}=a_{u k} \frac{E x_{U k}}{U_{k}} ; \quad \gamma_{c k}=a_{c k} \frac{E x_{C k}}{C_{k}}
\end{aligned}
$$

equations $(5.1 ; 5.5$; D.10; D.21; D.22) describe the damped harmonic oscillators:

$$
\begin{gathered}
{\left[\frac{d^{2}}{d t^{2}}+\gamma_{u k} \frac{d}{d t}+\omega_{u k}^{2}\right] u(\boldsymbol{k} ; t)=0 ; \quad\left[\frac{d^{2}}{d t^{2}}+\gamma_{c k} \frac{d}{d t}+\omega_{c k}^{2}\right] c(\boldsymbol{k} ; t)=0} \\
u(\boldsymbol{k} ; t) \sim \sin \varphi_{u k} t \exp -\frac{\gamma_{u k}}{2} t ; c(\boldsymbol{k} ; t) \sim \sin \varphi_{c k} t \exp -\frac{\gamma_{c k}}{2} t \\
\varphi_{u k}^{2}=\omega_{u k}^{2}-\frac{\gamma_{u k}^{2}}{4} ; \varphi_{c k}^{2}=\omega_{c k}^{2}-\frac{\gamma_{c k}^{2}}{4}
\end{gathered}
$$

If $\gamma_{u k}>0$ and $\gamma_{c k}>0$ than (D.25) and (D.4) describe the trade and the price $p(2 ; t)$ disturbances as the harmonic oscillations with dissipating amplitudes. But if $\gamma_{u k}<0$ or $\gamma_{c k}<0$ than (D.25) and $p(2 ; t)$ in (D.4) and the volatility (5.6) have harmonic modes with the exponentially growing amplitudes. It is interesting that the perturbations of the expectations $E x_{u}(\boldsymbol{k} ; t), E x_{c}(\boldsymbol{k} ; t)(\mathrm{D} .18)$ can generate the disturbances of the trades (D.25), the price $p(2 ; t)$ (D.4) and the volatility $\sigma_{p}{ }^{2}(t)(2.17 ; 5.6)$ like the harmonic oscillations with the amplitude growth by the exponent in time. It is obvious that such exponential growth of the perturbations $\pi(2 ; t)$ and the price volatility $\sigma_{p}^{2}(t)(2.17)$ is reduced by the applicability of the model equations $(5.1,5.5)$ and the relations (D.1, D.2, D.4, D.14, D.15, D.17). This approximation has sense till the perturbations remain small to compare with 1.

The relations (D.4, D.8) describe the mean square of the price $p(2 ; t)(3.14)$ disturbances. We derive the similar relations for the mean price $p(1 ; t)$ disturbances (Olkhov, 2019b):

$$
p(1 ; t)=p_{10}\left[1+\sum_{k, l=1}^{K}\left(\mu_{1 \boldsymbol{k}} c(1, \boldsymbol{k} ; t)-\lambda_{1 k} u(1, \boldsymbol{k} ; t)\right)\right]
$$

The coefficients $p_{10}, \mu_{l k}, \lambda_{l k}$ and the functions $c(1, \boldsymbol{k} ; t), u(1, \boldsymbol{k} ; t)(\mathrm{D} .26)$ describe the price $p(1 ; t)$ and the first-degree value and the volume in a way similar to $p_{10}, \mu_{l k}, \lambda_{l k}$ (D.4) and the functions $c(1, \boldsymbol{k} ; t), u(1, \boldsymbol{k} ; t)$ (D.5) for $p(2 ; t)$ (D.4) and the second-degree value and the volume (D.1). The disturbances of $p(2 ; t)(\mathrm{D} .4)$ and $p(1 ; t)$ (D.26) describe the price volatility $\sigma_{p}^{2}(t)(2.17)$ in the linear approximation by disturbances as:

$$
\begin{gathered}
\sigma_{p}^{2}(t)=\sigma_{p 0}^{2}\left\{1+\sum_{k, l=1}^{K} \sigma_{2}\left[\mu_{2 \boldsymbol{k}} c(2, \boldsymbol{k} ; t)-\lambda_{2 k} u(2, \boldsymbol{k} ; t)\right]-\sigma_{1}\left[\mu_{1 \boldsymbol{k}} c(1, \boldsymbol{k} ; t)-\lambda_{1 k} u(1, \boldsymbol{k} ; t)\right]\right\} \\
\sigma_{p 0}^{2}=p_{20}-p_{10}^{2} ; \sigma_{2}=\frac{p_{20}}{\sigma_{p 0}^{2}} ; \sigma_{1}=2 \frac{p_{10}^{2}}{\sigma_{p 0}^{2}}
\end{gathered}
$$

In (D.27) the constants and the functions with index 2 describe the price $p(2 ; t)(2.4 ; 2.7)$ for $n=2$ and its disturbances and the index 1 describes the price $p(1 ; t)(1.3)($ Olkhov, 2019b) and its disturbances. 